\title{
On robust interval observer design for uncertain systems subject to both time-invariant and time-varying uncertainties
}

\author{
Masoud Pourasghar, Vicenç Puig and Carlos Ocampo-Martinez \\ Automatic Control Department, Universitat Politècnica de Catalunya, Institut de Robòtica i \\ Informàtica Industrial (CSIC-UPC), Supervision, Safety and Automatic Control Research \\ Center (CS2AC), C/. Llorens i Artigas 4-6, 08028 Barcelona, Spain.
}

\section{ARTICLE HISTORY}

Compiled January 12, 2021

\begin{abstract}
This paper proposes the design of an interval observer-based approach for linear dynamic systems affected by both time-invariant and time-varying uncertainties. First, different interval observer schemes are compared and analyzed when dealing with the different type of uncertainties. Then, an integrated interval observer is proposed in order to overcome the drawbacks of using the set-based approach, i.e., the non-preservation of the parameter uncertainty time dependency and the wrapping effect. Furthermore, $H_{\infty}$ performance is considered in order to compute the observer gain by using an LMI technique. Finally, a numerical example and a real case study based on a two-tank system are employed for both illustrating and analyzing the effectiveness of the proposed approach.
\end{abstract}

\section{KEYWORDS}

Uncertain dynamic system, interval observers, time-invariant and time-varying uncertainties, LMI technique, $H_{\infty}$ performance

\section{Introduction}

Increasing the performance of an automatic control system as well as its safety and reliability has been important topics in the scientific community over the past years (Blanke, Kinnaert, Lunze, Staroswiecki, \& Schröder, 2006). In this regard, fault diagnosis of dynamic systems behavior plays a key role in the field of automatic control engineering (Chen \& Patton, 2012; Gertler, 1997). Generally speaking, there are two major classifications of fault diagnosis methods: i) model-based methods, ii) databased methods. In the former class, tracking the system behavior is done based on the mathematical model of the plant, e.g., observer-based approach, Kalman filter, extended Kalman filter, input-output and state-space based methods, while the latter class includes those methods that do not use the mathematical model for the same purpose, e.g., neural networks, pattern recognition or fuzzy logic approaches (Puig, Stancu, \& Quevedo, 2005b; Zhang \& Jiang, 2008).

Model-based approaches rely on the quality of the mathematical model describing the system behavior (Blanke et al., 2006; Puig, Montes de Oca, \& Blesa, 2013). However, a major problem with model-based approaches is related on how modelling 
uncertainties are considered (Alamo, Bravo, \& Camacho, 2005; Nam, Trinh, \& Pathirana, 2016; Pourasghar, Puig, Ocampo-Martinez, \& Zhang, 2017; Raïssi, Efimov, \& Zolghadri, 2012). The effect of uncertainties is the cause of the mismatch between the model and the real behavior of the system (Puig et al., 2013). Robust methods have recently been investigated using several approaches to explicitly consider such uncertainties in the context of fault diagnosis (Efimov, Raïssi, \& Zolghadri, 2013; Karimi Pour, Puig, \& Ocampo-Martinez, 2017; Puig et al., 2005b; Zhang \& Jiang, 2008). In this regard, a considerable amount of literature has been reported regarding different methods to model the effect of uncertainties, which can be categorized into stochastic and deterministic paradigms. Representing the uncertainty as a random variable is the main concept of stochastic approaches. One major drawback of these approaches is that the knowledge about the statistical distribution of the uncertainty should be available. On the other hand, the uncertainty in the deterministic approaches is considered unknown but bounded using several families of geometrical structures, e.g., interval boxes, polytopes, ellipsoids, and zonotopes, among others. Therefore, only the bounds of the uncertainty are required, which can be obtained based on the physical description of the considered system (Alamo et al., 2005; Combastel, 2015; Kalman, 1960; Maybeck, 1982; Schweppe, 1968).

In order to bound the uncertainty effect in the system using interval observers considering an unknown-but-bounded deterministic framework, there exist two main approaches: the set-based interval observer approach (Combastel, 2015; Nam, Pathirana, \& Trinh, 2014) and the trajectory-based interval observer approach (Puig et al., 2005b). In the set-based interval observer approaches, the set that bounds the outputs/states is determined using the observer equations based on previous approximated sets and using a one-step ahead prediction. On the other hand, for the trajectory-based interval observer approach, a set of point-wise trajectories generated by selecting particular values of the uncertainty is used (Combastel, 2015; Pourasghar et al., 2017; Puig et al., 2005b; Raïssi et al., 2012). Based on the literature, each approach has its own advantages and disadvantages. The set-based interval observer approach is affected by some problems, e.g., wrapping effect, range evaluation of an interval function (in this case, the state-space function) and the uncertain parameter time dependency (Puig et al., 2005b). However, in the second case, the interval hull of the state estimation is built following real trajectories generated by selecting particular values of the interval parameter vector. Consequently, this approach overcomes the wrapping effect and preserves the uncertain parameter time dependency, but in the case of the trajectory function, the problem of the interval function range evaluation still remains. On the other hand, set-based interval observer approaches present a lower computational complexity than trajectory-based interval observer approaches and, consequently, they seem to be more suitable for real-time applications (Kolev \& Petrakieva, 2005; Le, Alamo, Camacho, Stoica, \& Dumur, 2012).

According to Puig, Saludes, \& Quevedo (2003), it is possible to classify the approaches dealing with the time variance of the uncertain parameters into the timevarying approach and the time-invariant approach. Then, two approaches about the assumption of the time-variance of the uncertain parameters are possible:

- the time-varying approach, where uncertain parameters are unknown but bounded in their uncertainty intervals and can vary at each time step since one-step ahead recursion algorithms are used. This is the approach followed by El Ghaoui \& Calafiore (2000) and Puig, Cugueró, \& Quevedo (2001), among others. 
- the time-invariant approach, which assumes that uncertain parameters are unknown but bounded in their uncertainty intervals and guarantee that they cannot vary at each time step since a functional relation between parameters and states is used instead of a one-step ahead recursion. This is the approach followed by Horak (1988); Tibken (1993)n and Puig, Saludes, \& Quevedo (1999), among others.

Concerning the preservation of time dependency of uncertainty in the reported approaches from the literature, one possibility is to evolve the observer dynamics from the initial state to the present state by driving a functional relationship between states and parameters at every time instant (Puig et al., 2003) but with a high computational cost. To avoid such a complexity, the observer is usually designed to satisfy the monotonicity condition (Efimov, Raïssi, \& Zolghadri, 2013; Karimi Pour et al., 2017) such that only propagating some trajectories is enough to bound the effect of the uncertainty in the estimation provided by the observer.

When applying interval observers to fault detection, additionally to the problem of generating the detection thresholds by uncertainty propagation, another important problem is how to design the observer gain to be as robust as possible against the unavoidable effect of uncertainties. In this regard, there has been an increasing interest in computing the observer gain in several manners to minimize the effect of uncertainties. Thus, the observers can be designed by considering the robustness against disturbances, noise or any other uncertainties using, as e.g., $H_{\infty}$ optimization, Linear Matrix Inequalities (LMIs), among other strategies (Sadrnia, Chen, \& Patton, 1996; Zhong, Ding, Lam, \& Wang, 2003).

In the case of set-based approach, a robust observer interval design were reported by Wang, Zhou, Puig, Cembrano, \& Wang (2017) and Wang, Wang, Puig, \& Cembrano (2018) based on zonotopes for discrete-time uncertain systems. Moreover, in the case of trajectory-based observer, the design of an interval observer is done for discrete-time Linear Parameter-Varying (LPV) systems. However, in both cases, only the time-varying uncertainty is considered. In recent years, there has been an increasing interest in considering the time-invariant uncertainty (Pourasghar et al., 2017; Raïssi et al., 2012). The interval observer design has grown in importance and becomes more challenging in case the system is affected by both time-varying and time-invariant uncertainties. Therefore, more research on this topic needs to be undertaken considering both type of uncertainties in set-based and trajectory-based interval observer approaches. In this regard, the main contribution of this paper is focused on proposing a robust interval observer approach considering both time-varying and time-invariant uncertainties whose observer gain is computed by using LMI techniques to achieve $H_{\infty}$ performance, i.e., to be as robust as possible against the effect of uncertainty. Furthermore, the relationship between different interval observer approaches is discussed in order to propose an integrated approach taking into account both time-varying and time-invariant uncertainties. The effectiveness of the proposed approach is illustrated through a numerical example and a two-tank real system.

The remainder of the paper is organized as follows. The problem statement is addressed in Section 2, while the set-based and proposed interval observer structures and their robust design are discussed in Sections 3 and 4, respectively. The discussion about the whole proposed approach and a comparative assessment are presented in Section 5. Applications based on both a numerical example and a real two-tank system are used in order to illustrate the effectiveness of the proposed approach in Section 6. Finally, conclusions are drawn in Section 7. For completeness, some relevant definitions 
and properties are recalled in the Appendix.

\section{Notation}

Throughout this paper, $\mathbb{R}^{n}$ denotes the set of $n$-dimensional real numbers and $\oplus$ denotes the Minkowski sum. Matrices are written using capital letter, e.g., $A$, the calligraphic notation is used for denoting sets, e.g., $\mathcal{X}$, the transfer functions are highlighted using script font, e.g., $\mathscr{H},\|\cdot\|_{s}$ denotes the $s$-norm, absolute value is represented by $|$.$| and [\underline{x}, \bar{x}]$ is an interval with lower bound $\underline{x} \in \mathbb{R}^{n}$ and upper bound $\bar{x} \in \mathbb{R}^{n}$.

\section{Problem statement}

\subsection{Main problem formulation}

This paper considers that the uncertain system is represented by a discrete-time linear time-invariant model in state-space form as follows:

$$
\begin{aligned}
x_{k+1} & =\left[A_{0}+\Delta A(\theta)\right] x_{k}+B u_{k}+E_{\omega} \omega_{k}, \\
y_{k} & =C x_{k}+E_{v} v_{k}
\end{aligned}
$$

where $u \in \mathbb{R}^{n_{u}}, y \in \mathbb{R}^{n_{y}}$ and $x \in \mathbb{R}^{n_{x}}$ are the input, the output and the state vectors, respectively. Moreover, $A_{0} \in \mathbb{R}^{n_{x} \times n_{x}}, B \in \mathbb{R}^{n_{x} \times n_{u}}$ and $C \in \mathbb{R}^{n_{y} \times n_{x}}$ are the state-space matrices. Both state disturbance and process noise vectors are considered as timevarying uncertainties and defined by $\omega \in \mathbb{R}^{n_{\omega}}$ and $v \in \mathbb{R}^{n_{v}}$, respectively. Moreover, $E_{\omega}$ and $E_{v}$ are the associated distribution matrices with appropriate dimensions while $k \in \mathbb{N}$ indicates the discrete time. Furthermore, it is assumed that the vector of timeinvariant uncertain parameters $\theta$ belongs to an admissible set $\Theta$, i.e.,

$$
\Theta=\left\{\theta \in \mathbb{R}^{n_{\theta}}: \underline{\theta}_{i} \leq \theta_{i} \leq \bar{\theta}_{i} \forall i=1, \ldots, n_{\theta}\right\},
$$

where $n_{\theta}$ denotes the number of uncertain parameters. Moreover, the matrix $A_{0}$ contains the nominal values of the parameters while $\Delta A(\theta)$ represents the related uncertainty.

Assumption 2.1. It is assumed that, for all $\theta \in \Theta$,

$$
\underline{\Delta A}<\Delta A(\theta)<\overline{\Delta A}
$$

where $\underline{\Delta A} \in \mathbb{R}^{n_{x} \times n_{x}}$ and $\overline{\Delta A} \in \mathbb{R}^{n_{x} \times n_{x}}$ are constant and known matrices that contain the minimum and maximum values of $\Delta A(\theta)$, respectively.

Additionally, the additive uncertainties, i.e., time-varying measurement disturbance $\omega$ and process noise $v$, are assumed unknown but bounded, i.e.,

$$
\begin{aligned}
\mathcal{W} & =\left\{\omega_{k} \in \mathbb{R}^{n_{\omega}}:\left|\omega_{k}-c_{\omega}\right| \leq \bar{\omega}, c_{\omega} \in \mathbb{R}^{n_{\omega}}, \bar{\omega} \in \mathbb{R}^{n_{\omega}}\right\} \\
\mathcal{V} & =\left\{v_{k} \in \mathbb{R}^{n_{v}}:\left|v_{k}-c_{v}\right| \leq \bar{v}, c_{v} \in \mathbb{R}^{n_{v}}, \bar{v} \in \mathbb{R}^{n_{v}}\right\}
\end{aligned}
$$

where $c_{\omega}, \bar{\omega}, c_{v}$ and $\bar{v}$ are vectors of constant entries. 
Remark 2.1. Note that the inequalities in Assumption 2.1 and (4) are considered component-wise.

\subsection{General observer structure}

Monitoring the system behavior with the dynamical model (1) can be done by designing a Luenberger observer of the form

$$
\begin{aligned}
\hat{x}_{k+1} & =A_{0} \hat{x}_{k}+B u_{k}+L\left(y_{k}-\hat{y}_{k}\right), \\
\hat{y}_{k} & =C \hat{x}_{k},
\end{aligned}
$$

where $\hat{x} \in \mathbb{R}^{n_{x}}$ and $\hat{y} \in \mathbb{R}^{n_{y}}$ are the state estimation and the output prediction, respectively. Furthermore, $L \in \mathbb{R}^{n_{x} \times n_{y}}$ denotes the observer gain that should be chosen such that $\left(A_{0}-L C\right)$ was a Schur matrix. Moreover, the pair $\left(A_{0}, C\right)$ is assumed to be detectable.

To take into account the effects of time-varying uncertainties, i.e., $\omega$ and $v$, and timeinvariant uncertainties, i.e., $\Delta A(\theta)$, over the output/state estimation provided by (5), two different strategies are described next: one based on bounding the uncertainty effect in the observer estimation and the other based on designing the observer gain $L$ to minimize such effect.

\section{Set-based observer}

\subsection{Set-based observer structure}

In the set-based observer approach, the underlying observer structure is determined using the algorithm proposed by Montes de Oca, Puig, \& Blesa (2012). Generally speaking, in this approach, the set of states at time instant $k+1$ is approximated by using propagation algorithms from the set of states at time $k$ (for more information see Puig, Stancu, \& Quevedo (2005a) and Combastel (2015)). Moreover, the gain matrix $L$ can be further tuned with respect to the state estimation purpose, i.e., to increase the robustness of the state estimation.

As mentioned before, the effect of uncertainty can be expressed using a zonotopicset representation, i.e., a particular type of polytope, reducing the set operations to simple matrix calculations. In this regard, the zonotopic representation of $\omega$ and $v$ in (4) can be written as

$$
\begin{gathered}
\mathcal{W}=\left\langle c_{\omega}, R_{\omega}\right\rangle, \\
\mathcal{V}=\left\langle c_{v}, R_{v}\right\rangle,
\end{gathered}
$$

where $c_{\omega}$ and $c_{v}$ denote the centers of the sets $\mathcal{W}$ and $\mathcal{V}$, respectively, with their generator matrices $R_{\omega} \in \mathbb{R}^{n_{\omega} \times n_{\omega}}$ and $R_{v} \in \mathbb{R}^{n_{v} \times n_{v}}$. Then, monitoring the dynamical system with a mathematical model as in (1) can be done by designing a Luenberger observer of the form (5) and following Proposition 3.1.

Assumption 3.1. The time-varying additive uncertainties represented in (6) are assumed to be bounded by a unit hypercube expressed as the centered zonotopes, i.e., 
$\forall k \geq 0, \omega_{k} \in[-1,1]^{n_{\omega}}=\left\langle 0, I_{n_{\omega}}\right\rangle, v_{k} \in[-1,1]^{n_{v}}=\left\langle 0, I_{n_{v}}\right\rangle$ where $I_{n_{\omega}}$ and $I_{n_{v}}$ denote identity matrices of suitable dimensions.

Assumption 3.2. The initial state $x_{0}$ belongs to the zonotopic set $\mathcal{X}_{0}=\left\langle c_{0}, R_{0}\right\rangle$, where $c_{0} \in \mathbb{R}^{n_{x}}$ denotes the center and $R_{0} \in \mathbb{R}^{n_{x} \times r_{R_{0}}}$ is non-empty matrix containing the generators matrix of the initial zonotope $\mathcal{X}_{0}$.

Proposition 3.1. (Zonotopic-observer structure) Considering the observer scheme (5) and the uncertainties modelled as in Assumptions 2.1, 3.1 and 3.2, the center c and the segment matrix $R$ of the zonotope that bounds the state estimation provided by the observer (5) can be recursively defined as

$$
\begin{aligned}
c_{k+1} & =\operatorname{mid}\left(A^{*}(\theta)\right) c_{k}+B u_{k}+L y_{k}, \\
R_{k+1} & =\left[\begin{array}{llll}
\left.\diamond A^{*}(\theta) \check{R}_{k}\right) & \frac{\operatorname{diam}\left(A^{*}(\theta)\right)}{2} c_{k} & E_{\omega} & -L E_{v}
\end{array}\right],
\end{aligned}
$$

where $A^{*}(\theta)=\left[A_{0}+\Delta A(\theta)\right]-L C, \check{R}_{k}=\downarrow_{q}\left\{R_{k}\right\}$ (see Property 2 in the Appendix), mid denotes the center and diam is the diameter of the interval. Moreover, the state inclusion property $\hat{x}_{k} \in\left\langle c_{k}, R_{k}\right\rangle$ holds for all $k \geq 0$ (see Properties 3 and 4 in the Appendix) make use of a zonotope inclusion $\diamond(\mathcal{Z})$ operator.

Proof. By including in the observer scheme (5) the uncertainties modelled as in Assumptions 2.1, 3.1 and 3.2, i.e.,

$$
\begin{aligned}
\hat{x}_{k+1} & =A^{*}(\theta) \hat{x}_{k}+B u_{k}+E_{\omega} \omega_{k}+L\left(y_{k}-\hat{y}_{k}\right), \\
\hat{y}_{k} & =C \hat{x}_{k}+E_{v} v_{k},
\end{aligned}
$$

and assuming that $\hat{x}_{k} \in\left\langle c_{k}, R_{k}\right\rangle, \omega_{k} \in\left\langle 0, I_{n_{\omega}}\right\rangle$ and $v_{k} \in\left\langle 0, I_{n_{v}}\right\rangle$, where the inclusion property is preserved by using the reduction operator, which means $\hat{x}_{k} \in\left\langle c_{k}, \check{R}_{k}\right\rangle$, then the the center $c$ and the segment matrix $R$ of the zonotopic representation of the interval observer can be written using (5) as follows:

$$
\begin{aligned}
c_{k+1} & =\left(A^{*}(\theta)\right) c_{k}+B u_{k}+L y_{k}, \\
R_{k+1} & =\left[\begin{array}{lll}
\left(A^{*}(\theta)\right) \check{R}_{k} & E_{\omega} & -L E_{v}
\end{array}\right] .
\end{aligned}
$$

Thus, based on Definition 2 and Properties 1 and 4 in the Appendix, $c_{k+1}$ and $R_{k+1}$ in (9) can be derived as in (7).

Both time-varying and time-invariant uncertainties are considered unknown but bounded in their uncertainty intervals and can vary arbitrarily at each time instant within the interval obtained using the zonotopic observer approach and Definition 5 in the Appendix.

\subsection{Robust set-based observer design}

In order to reduce the effect of uncertainties on the state estimation and achieving the accurate estimation, the well-known $H_{\infty}$ technique is used in this paper (Ding, 2008).

In this regard, considering Lemma 2, and according to Chen \& Patton (2012), the uncertain parameter in (1a) can be approximated only based on uncertain term. Then, 
(1a) can be written as

$$
x_{k+1}=A_{0} x_{k}+B u_{k}+E_{\omega} \omega_{k}+E_{\theta} \theta_{k}
$$

with

$$
\Delta A(\theta) x_{k} \approx E_{\theta} \theta_{k}
$$

where $\theta_{k} \in \mathbb{R}^{n_{\theta}}$ is a disturbance, namely, an unknown but constant vector. Moreover, $E_{\theta}$ is the associated non-empty distribution matrix of suitable dimensions that shows the direction of the uncertainty. It is worth mentioning that based on (79) in Lemma 2 the effect of the state is embedded in $\theta_{k}$.

Keeping these considerations in mind, the zonotopic observer structure in Proposition 3.1 can be rewritten by using Assumption 3.3 and Proposition 3.2.

Assumption 3.3. The time-invariant additive uncertainties represented in (11) are assumed to be bounded by a unit hypercube expressed as centered zonotope, i.e., $\forall k \geq 0, \theta_{k} \in[-1,1]^{n_{\theta}}=\left\langle 0, I_{n_{\theta}}\right\rangle$, where $I_{n_{\theta}}$ denotes the identity matrix.

Proposition 3.2. Considering the observer (5) and Assumptions 2.1, 3.1 and 3.2, the center $c$ and the shape matrix $R$ of the zonotope bounding the observer estimation can be recursively defined as

$$
\begin{aligned}
c_{k+1} & =\left(A_{0}-L C\right) c_{k}+B u_{k}+L y_{k}, \\
R_{k+1} & =\left[\begin{array}{llll}
\left(A_{0}-L C\right) \check{R}_{k} & E_{\omega} & -L E_{v} & E_{\theta}
\end{array}\right] .
\end{aligned}
$$

Proof. Assume $x_{k} \in\left\langle c_{k}, R_{k}\right\rangle, \omega_{k} \in\left\langle 0, I_{n_{\omega}}\right\rangle, v_{k} \in\left\langle 0, I_{n_{v}}\right\rangle$ and $\theta \in\left\langle 0, I_{n_{\theta}}\right\rangle$ for all $k \geq 0$, where the inclusion property is preserved and (5) can be written using the reduction operator as

$$
\begin{aligned}
x_{k+1} \in\left\langle c_{k+1}, R_{k+1}\right\rangle= & \left\langle\left(A_{0}-L C\right) c_{k},\left(A_{0}-L C\right) \check{R}_{k}\right\rangle \oplus\left\langle B u_{k}, 0\right\rangle \\
& \oplus\left\langle L y_{k}, 0\right\rangle \oplus\left\langle 0, E_{w}\right\rangle \oplus\left\langle 0,-L E_{v}\right\rangle \oplus\left\langle 0, E_{\theta}\right\rangle .
\end{aligned}
$$

Thus, based on Definition 2 and Property 1 , the center $c_{k+1}$ and the shape matrix $R_{k+1}$ in (13) can be expressed as in (12).

Now, the dynamics of estimation error using observer (5) and system uncertainty modelling (10) are introduced in Proposition 3.3.

Proposition 3.3. Given that the observation error in the set-based observer approach is defined as

$$
e_{k}=x_{k}-\hat{x}_{k},
$$

then, considering the dynamical model (1) and the observer structure (5), the dynamics of observation error can be obtained as

$$
e_{k+1}=\left(A_{0}-L C\right) e_{k}+E_{d} d_{k}
$$


where

$$
\begin{aligned}
E_{d} & =\left[\begin{array}{lll}
E_{\theta} & E_{\omega} & -L E_{v}
\end{array}\right], \\
d_{k} & =\left[\begin{array}{lll}
\theta_{k} & \omega_{k} & v_{k}
\end{array}\right]^{\top} .
\end{aligned}
$$

Proof. Based on Lemma 2 in Appendix, (1a) can be rewritten as in (10). Therefore, considering the state estimation error as in (14), the dynamics of the observation error can be obtained using (1), (10), (5) and (14), yielding to (15).

Considering the transfer function $\mathscr{G}_{e d}(z)$ from uncertainties to the state estimation error, where $z$ denotes the $z$-transform, the $H_{\infty}$ norm of $\mathscr{G}_{e d}(z)$ is known as the maximum singular value of $\mathscr{G}_{e d}(z)$. Then, according to Ding (2008), Theorem 3.4 can be used to compute the observer gain minimizing the effect of the uncertainty and leading to a robust observer.

Theorem 3.4. Given a scalar $\gamma>0$, the state estimation error dynamics in (15) are stable and satisfy the following $H_{\infty}$ performance index:

$$
\left\|\mathscr{G}_{e d}(z)\right\|_{\infty}<\gamma,
$$

if there exists a symmetric positive definite matrix $P \in \mathbb{R}^{n_{x} \times n_{x}}$, i.e., $P>0$, and a matrix $M \in \mathbb{R}^{n_{x} \times n_{y}}$ such that

$$
\left[\begin{array}{cccccc}
-P & P A_{0}-M C & P E_{\theta} & P E_{\omega} & -M E_{v} & 0 \\
* & -P & 0 & 0 & 0 & I \\
* & * & \gamma I & 0 & 0 & 0 \\
* & * & * & \gamma I & 0 & 0 \\
* & * & * & * & \gamma I & 0 \\
* & * & * & * & * & \gamma I
\end{array}\right]<0 .
$$

In the case that the LMI (19) can be solved, the gain of the observer can be computed as

$$
L=P^{-1} M .
$$

Proof. Considering the Proposition 3.3, the transfer function $\mathscr{G}_{e d}(z)$ can be obtained as

$$
\mathscr{G}_{e d}(z)=\left(z I-\left(A_{0}-L C\right)\right)^{-1} E_{d} .
$$

Then, according to Ding (2008), it can be written that $\left(A_{0}-L C\right)$ is a stable matrix and $\left\|\left(z I-\left(A_{0}-L C\right)\right)^{-1} E_{d}\right\|<\gamma$. Furthermore, there exists a symmetric positive definite $P$ such that

$$
\left[\begin{array}{cccc}
-P & P\left(A_{0}-L C\right) & P E_{d} & 0 \\
\left(A_{0}-L C\right)^{\top} P & -P & 0 & I \\
E_{d}^{\top} P & 0 & \gamma I & 0 \\
0 & I & 0 & \gamma I
\end{array}\right]<0 .
$$


Now, by substituting (16) into (22) and using the Schur complement, (22) can be rewritten as

$$
\left[\begin{array}{cccccc}
-P & P\left(A_{0}-L C\right) & P E_{\theta} & P E_{\omega} & -P L E_{v} & 0 \\
* & -P & 0 & 0 & 0 & I \\
* & * & \gamma I & 0 & 0 & 0 \\
* & * & * & \gamma I & 0 & 0 \\
* & * & * & * & \gamma I & 0 \\
* & * & * & * & * & \gamma I
\end{array}\right]<0 .
$$

Now, by introducing the new variable $M=P L$, the LMI in (19) can be obtained.

\subsection{Guaranteed state estimation using an optimization-based method}

Based on Definition 4 in the Appendix, the size of the zonotope in (12), measured by W-radius of a zonotope, e.g., $\mathcal{S}=\langle c, R\rangle$ with $R \in \mathbf{B}^{n_{s}}$ where $\mathbf{B}=[-1,1]$ is a hypercube with proper dimension, is defined as $\iota_{w}$, and it is computed using

$$
\begin{aligned}
\iota_{w, k+1} & =\max _{\stackrel{\circ}{s}_{k+1} \in \mathbf{B}^{\left(n_{s}+n_{x}+2 n_{y}\right)}}\left\|R_{k+1} \stackrel{\circ}{s+1}_{k+1}\right\|_{2, W}^{2} \\
& =\max _{\stackrel{\circ}{s}_{k+1} \in \mathbf{B}^{\left(n_{s}+n_{x}+2 n_{y}\right)}} \stackrel{\circ}{s}_{k+1}^{\top} R_{k+1}^{\top} W R_{k+1} \stackrel{\circ}{s+1}_{k+1},
\end{aligned}
$$

where $\stackrel{\circ}{s} \in \mathbf{B}^{n_{s}}$ is a unitary box and $W$ is a weighting matrix.

Then, the gain of the observer can be obtained by minimizing the size of the statebounding zonotope as in Theorem 3.5. Acá vuelven a aparecer las "s" con bolitas. ¿Son necesarias?

Theorem 3.5. Consider that the state-bounding zonotope $\hat{\mathcal{X}}$ in Proposition 3.2 is parametrized by means of the observer gain, i.e., $\hat{\mathcal{X}}_{k+1}(L)=\left\langle c_{k+1}(L), R_{k+1}(L)\right\rangle$. Then, considering $\rho \in(0,1)$ and $\epsilon>0$, the minimization criterion of the size of the zonotope $\hat{\mathcal{X}}$, i.e.,

$$
\iota_{w, k+1} \leq \rho \iota_{w, k}+\epsilon
$$

holds if there exist matrices $W \in \mathbb{R}^{n_{x} \times n_{x}}, W=W^{\top}>0, Y \in \mathbb{R}^{n_{x} \times n_{y}}$, and diagonal matrices $\Gamma \in \mathbb{R}^{n_{x} \times n_{x}}, \Upsilon \in \mathbb{R}^{n_{y} \times n_{y}}$, and $\Omega \in \mathbb{R}^{n_{x} \times n_{x}}$ such that

$$
\begin{aligned}
& {\left[\begin{array}{ccccc}
\rho W & * & * & * & * \\
0 & \Gamma & * & * & * \\
0 & 0 & \Upsilon & * & * \\
0 & 0 & 0 & \Omega & * \\
W A_{0}-Y C & W E_{\omega} & -Y E_{v} & W E_{\theta} & W
\end{array}\right]>0,} \\
& \Gamma>0, \Upsilon>0, \Omega>0, \\
& \operatorname{tr}(\Gamma)+\operatorname{tr}(\Upsilon)+\operatorname{tr}(\Omega)<\epsilon
\end{aligned}
$$

are satisfied. 
Proof. Considering (24) and (25), it follows that

$$
\max _{\stackrel{\circ}{s}_{k+1} \in \mathbf{B}^{\left(n_{s}+n_{\omega}+2 n_{v}\right)}}\left\|R_{k+1} \stackrel{\circ}{s}_{k+1}\right\|_{2, W}^{2}-\max _{\dot{s}_{k} \in \mathbf{B}^{n_{s}}} \rho\left\|R_{k} \stackrel{\circ}{s}_{k}\right\|_{2, W}^{2}-\epsilon \leq 0 .
$$

Then, considering that for any $s_{k} \in \mathbf{B}^{n_{s}}$,

$$
\max _{s_{k} \in \mathbf{B}^{n_{s}}}\left\|R_{k} \stackrel{\circ}{s}_{k}\right\|_{2, W}^{2} \geq\left\|R_{k} s_{k}\right\|_{2, W}^{2}
$$

the sufficient condition

$$
\left\|R_{k+1} s_{k+1}\right\|_{2, W}^{2}-\rho\left\|R_{k} s_{k}\right\|_{2, W}^{2}-\epsilon<0
$$

holds.

Furthermore, recalling the shape matrix of the state-bounding zonotope in (12) and setting $Y=W L$, and also denoting

$$
R_{k+1}=\left[\begin{array}{llll}
W A_{0}-Y C & W E_{\omega} & -Y E_{v} & W E_{\theta}
\end{array}\right]
$$

then, (29) can be rewritten as

$$
\Pi^{\top} R_{k+1}^{\top} W^{-1} R_{k+1} \Pi-\rho s_{k}^{\top} R_{k}^{\top} W R_{k} s_{k}-\epsilon<0,
$$

where $\Pi=\left[\begin{array}{c}R_{k} s_{k} \\ \alpha_{1} \\ \alpha_{2} \\ \alpha_{3}\end{array}\right]$. Thus, for any diagonal positive semi-definite matrices $\Gamma, \Upsilon$ and $\Omega$, then, it can be written for any $\alpha_{1} \in \mathbf{B}^{n_{x}}, \alpha_{2} \in \mathbf{B}^{n_{y}}$ and $\alpha_{3} \in \mathbf{B}^{n_{y}}$ that

$$
\begin{aligned}
& \alpha_{1}^{\top} \Gamma \alpha_{1}=\sum_{i=1}^{n_{x}} \alpha_{1}^{2} \Gamma_{i} \leq \operatorname{tr}(\Gamma), \\
& \alpha_{2}^{\top} \Upsilon \alpha_{2}=\sum_{i=1}^{n_{y}} \alpha_{2}^{2} \Upsilon_{i} \leq \operatorname{tr}(\Upsilon), \\
& \alpha_{3}^{\top} \Omega \alpha_{3}=\sum_{i=1}^{n_{y}} \alpha_{3}^{2} \Omega_{i} \leq \operatorname{tr}(\Omega),
\end{aligned}
$$

where $\Gamma_{i}, \Upsilon_{i}$ and $\Omega_{i}$ are the diagonal elements of $\Gamma, \Upsilon$ and $\Omega$, respectively. Therefore, using (32), it can be obtained that

$$
\begin{aligned}
& \operatorname{tr}(\Gamma)-\alpha_{1}^{\top} \Gamma \alpha_{1} \geq 0, \quad \forall \alpha_{1} \in \mathbf{B}^{n_{x}}, \\
& \operatorname{tr}(\Upsilon)-\alpha_{2}^{\top} \Upsilon \alpha_{2} \geq 0, \quad \forall \alpha_{2} \in \mathbf{B}^{n_{y}}, \\
& \operatorname{tr}(\Omega)-\alpha_{3}^{\top} \Omega \alpha_{3} \geq 0, \quad \forall \alpha_{3} \in \mathbf{B}^{n_{y}} .
\end{aligned}
$$


Thus, a sufficient condition of (31) can be obtained by adding (33) to (31) as

$$
\begin{aligned}
& \Pi^{\top} R_{k+1}^{\top} W^{-1} R_{k+1} \Pi-\rho s_{k}^{\top} R_{k}^{\top} W R_{k} s_{k}+\operatorname{tr}(\Gamma)-\alpha_{1}^{\top} \Gamma \alpha_{1}+\operatorname{tr}(\Upsilon) \\
& \quad-\alpha_{2}^{\top} \Upsilon \alpha_{2}+\operatorname{tr}(\Omega)-\alpha_{3}^{\top} \Omega \alpha_{3}-\epsilon<0 .
\end{aligned}
$$

Moreover, (34) can be rearranged as

$$
\begin{aligned}
& \left(\Pi^{\top} R_{k+1}^{\top} W^{-1} R_{k+1} \Pi-\rho s_{k}^{\top} R_{k}^{\top} W R_{k} s_{k}-\alpha_{1}^{\top} \Gamma \alpha_{1}-\alpha_{2}^{\top} \Upsilon \alpha_{2}-\alpha_{3}^{\top} \Omega \alpha_{3}\right)+ \\
& \quad(\operatorname{tr}(\Gamma)+\operatorname{tr}(\Upsilon)+\operatorname{tr}(\Omega)-\epsilon)<0 .
\end{aligned}
$$

If (26c) holds, the satisfaction of (34) can be guaranteed when

$$
\left(\Pi^{\top} R_{k+1}^{\top} W^{-1} R_{k+1} \Pi-\rho s_{k}^{\top} R_{k}^{\top} W R_{k} s_{k}-\alpha_{1}^{\top} \Gamma \alpha_{1}-\alpha_{2}^{\top} \Upsilon \alpha_{2}-\alpha_{3}^{\top} \Omega \alpha_{3}\right)<0,
$$

that can be reformulated as

$$
\Pi^{\top}\left(R_{k}^{\top} W^{-1} R_{k}-\left[\begin{array}{cccc}
\rho W & 0 & 0 & 0 \\
0 & \Gamma & 0 & 0 \\
0 & 0 & \Upsilon & 0 \\
0 & 0 & 0 & \Omega
\end{array}\right]\right) \Pi<0 .
$$

Moreover, from (35), the sufficient condition

$$
\left[\begin{array}{cccc}
\rho W & 0 & 0 & 0 \\
0 & \Gamma & 0 & 0 \\
0 & 0 & \Upsilon & 0 \\
0 & 0 & 0 & \Omega
\end{array}\right]-R_{k}^{\top} W^{-1} R_{k}>0
$$

is obtained. Now, using the Schur complement and considering (30), the LMI in (26a) is then obtained.

Algorithm 1 summarizes the state estimation methodology using the set-based interval observer approach.

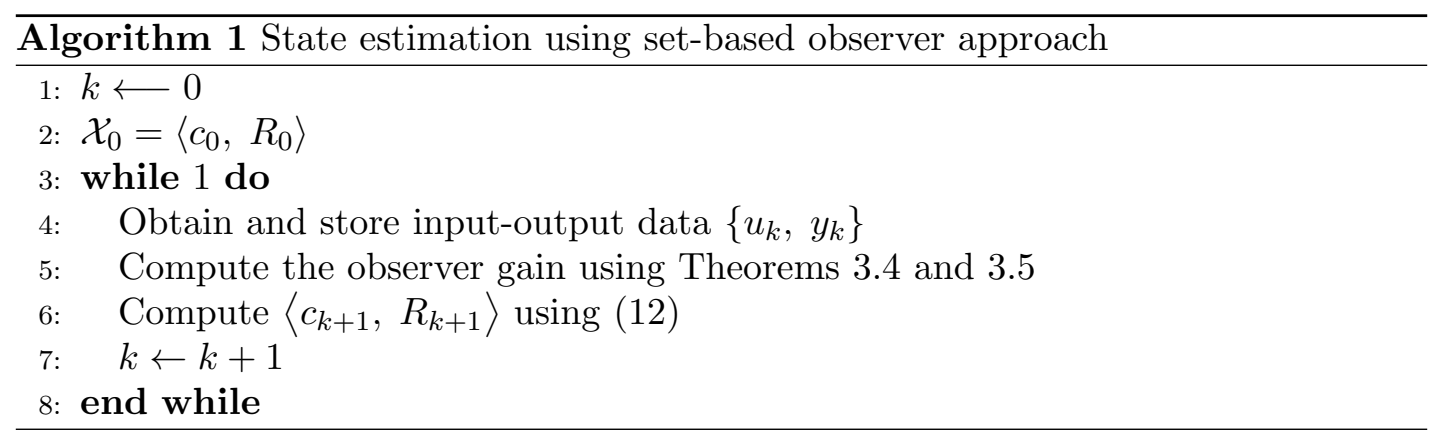

In order to overcome the problems associated to the set-based interval observer approach, e.g., wrapping effect and range evaluation of an interval function, already discussed in Section 1, the state estimation can be computed using the trajectorybased interval observer approach that relies on the computation of the approximated state set using point-wise trajectories. A discussion of such approach will be the main topic of the next section. 


\section{Interval observer approach}

\subsection{Interval observer structure}

RAE-1

As mentioned before, in the case of trajectory-based interval observer approach, the value of parameter uncertainty is unknown but bounded within an interval and its invariance can be guaranteed at each time instant. In this approach, the interval of the states can be estimated at each iteration by using specific state trajectories corresponding to particular values of uncertainties $\Delta A(\theta)$.

According to Puig et al. (2003), the loss of the time dependency of the parametric uncertainty in the set-based interval observer approach and the problem of wrapping effect can be avoided by deriving a function based on the relationship between the states and parameters from the initial state to the current state by considering the observer dynamics including uncertainties (8) as

$$
\hat{x}_{k}=\left(A^{*}(\theta)\right)^{k} x_{0}+\sum_{j=0}^{k-1}\left(A^{*}(\theta)\right)^{k-1-j} B^{*} u_{j}^{*},
$$

where

$$
B^{*}=\left[\begin{array}{llll}
B & L & E_{\omega} & -L E_{v}
\end{array}\right], \quad u^{*}=\left[\begin{array}{llll}
u_{k} & y_{k} & \omega_{k} & v_{k}
\end{array}\right]^{\top} .
$$

Then, considering $\theta \in \Theta$, both upper and lower bounds of the state estimation of the dynamical model $(1)$, i.e., $\hat{\mathcal{X}}(k)=[\underline{\hat{x}}(k), \overline{\hat{x}}(k)]$, can be obtained by solving the following optimization problems:

$$
\begin{aligned}
& \overline{\hat{x}}_{k}=\max _{\theta \in \Theta, \omega_{j} \in \mathcal{W}, v_{j} \in \mathcal{V}}\left[\left(A^{*}(\theta)\right)^{k} \hat{x}_{0}+\sum_{j=0}^{k-1}\left(A^{*}(\theta)\right)^{k-1-j} B^{*} u_{j}^{*}\right], \\
& \underline{\hat{x}}_{k}=\min _{\theta \in \Theta, \omega_{j} \in \mathcal{W}, v_{j} \in \mathcal{V}}\left[\left(A^{*}(\theta)\right)^{k} \hat{x}_{0}+\sum_{j=0}^{k-1}\left(A^{*}(\theta)\right)^{k-1-j} B^{*} u_{j}^{*}\right],
\end{aligned}
$$

both subject to

$$
x_{0} \in \square \mathcal{X}_{0},
$$

where $\underline{\hat{x}}_{k}$ and $\overline{\hat{x}}_{k}$ denote the lower and upper bounds of the interval approximation, respectively.

Remark 4.1. It is worth mentioning that both upper and lower bounds of the interval approximation of $\hat{x}_{k}$ should be obtained separately for each component.

Numerical methods can be used to solve the optimization problems in (38) for computing $\overline{\hat{x}}$ and $\underline{\hat{x}}^{1}$. However, the computational burden is high. Alternatively, when designing the observer to result in a monotonic system as in Efimov, Raïssi, Perruquetti, \& Zolghadri (2013), the solution of (38) is achieved using the extreme values of uncertainties. This means that just considering two different observers, one per each extreme value for estimating the upper and lower bounds, is enough for reduc-

\footnotetext{
${ }^{1}$ This approach is deeply investigated in Puig et al. (2003).
} 
ing the computational load. These proposed observer design can be done by following Proposition 4.1.

Proposition 4.1. Considering an observer that satisfies the monotonicity property, the time-invariant uncertainty $\theta \in \Theta$, Lemma 1 and Assumption 2.1, the numerical solution of (38) is achieved using the structure of the interval observer approach with the purpose of estimating the state as

$$
\begin{aligned}
& \overline{x_{k+1}}=\left(A_{0}-\bar{L} C\right) \overline{x_{k}}+B u_{k}+\bar{L} y_{k}+\overline{E_{d}} \overline{d_{k}}+\overline{\xi_{k}}, \\
& \underline{x_{k+1}}=\left(A_{0}-\underline{L} C\right) \underline{x_{k}}+B u_{k}+\underline{L} y_{k}+\underline{E_{d}} \underline{d_{k}}+\underline{\xi_{k}},
\end{aligned}
$$

with

$$
\begin{aligned}
& \overline{E_{d}}=\left[\begin{array}{ll}
E_{\omega} & -\bar{L} E_{v}
\end{array}\right], \quad \overline{d_{k}}=\left[\begin{array}{ll}
\overline{\omega_{k}} & \overline{v_{k}}
\end{array}\right]^{\top}, \\
& \underline{E_{d}}=\left[\begin{array}{ll}
E_{\omega} & -\underline{L} E_{v}
\end{array}\right], \quad \underline{d_{k}}=\left[\begin{array}{ll}
\omega_{k} & \underline{v_{k}}
\end{array}\right]^{\top}, \\
& \overline{\xi_{k}}=\overline{\Delta A^{+}} \overline{x_{k}^{+}}-\underline{\Delta A^{+}} \overline{x_{k}^{-}}-\overline{\Delta A^{-}} \underline{x_{k}^{+}}+\underline{\Delta A^{-}} \underline{x_{k}^{-}}, \\
& \underline{\xi_{k}}=\underline{\Delta A^{+}} \underline{x_{k}^{+}}-\overline{\Delta A^{+}} \underline{x_{k}^{-}}-\underline{\Delta A^{-} \overline{x_{k}^{+}}}+\overline{\Delta A^{-}} \overline{x_{k}^{-}}, \\
& \overline{\Delta A^{+}}=\max \{0, \overline{\Delta A}\}, \quad \overline{\Delta A^{-}}=\overline{\Delta A^{+}}-\overline{\Delta A}, \\
& \underline{\Delta A^{+}}=\max \{0, \underline{\Delta A}\}, \quad \underline{\Delta A^{-}}=\underline{\Delta A^{+}}-\underline{\Delta A}, \\
& \overline{x^{+}}=\max \{0, \bar{x}\}, \quad \overline{x^{-}}=\overline{x^{+}}-\bar{x}, \\
& \underline{x^{+}}=\max \{0, \underline{x}\}, \quad \underline{x^{-}}=\underline{x^{+}}-\underline{x},
\end{aligned}
$$

where $\bar{L} \in \mathbb{R}^{n_{x} \times n_{y}}$ and $\underline{L} \in \mathbb{R}^{n_{x} \times n_{y}}$ are the gains of upper and lower observers, respectively.

Proof. Considering the satisfaction of monotonicity property for the observer, (37) can be unfolded as

$$
\hat{x}_{k}=\left(A^{*}(\theta)\right)^{k} x_{0}+\sum_{j=0}^{k-1}\left(A^{*}(\theta)\right)^{k-1-j}\left(B u_{k, j}+L y_{k, j}+E_{\omega} \omega_{k, j}-L E_{v} v_{k, j}\right) .
$$

It can be seen from (41) that the estimation depends on $\theta, \omega$ and $v$. Moreover, as it is proposed by Puig et al. (2003), concerning the trajectory-based approach used to compute a estimation of the system state region, the observer state region $\hat{\mathcal{X}}_{k}$ will be bounded at any time instant $k$ by its interval hull $\square \hat{\mathcal{X}}_{k+1}=\left[\underline{\hat{x}}_{k}, \overline{\hat{x}}_{k}\right]$ where

$$
\begin{aligned}
\overline{\hat{x}}_{k}= & \max _{\theta \in \Theta, \omega_{j} \in \mathcal{W}, v_{j} \in \mathcal{V}}\left[\left(A^{*}(\theta)\right)^{k} x_{0}\right. \\
& \left.+\sum_{j=0}^{k-1}\left(A^{*}(\theta)\right)^{k-1-j}\left(B u_{k, j}+\bar{L} y_{k, j}+E_{\omega} \omega_{k, j}-\bar{L} E_{v} v_{k, j}\right)\right], \\
\underline{\hat{x}}_{k}= & \min _{\theta \in \Theta, \omega_{j} \in \mathcal{W}, v_{j} \in \mathcal{V}}\left[\left(A^{*}(\theta)\right)^{k} x_{0}\right. \\
& \left.+\sum_{j=0}^{k-1}\left(A^{*}(\theta)\right)^{k-1-j}\left(B u_{k, j}+\underline{L} y_{k, j}+E_{\omega} \omega_{k, j}-\underline{L} E_{v} v_{k, j}\right)\right],
\end{aligned}
$$


both subject to

$$
x_{0} \in \square \mathcal{X}_{0},
$$

and assuming time-invariant uncertain parameters (this method is known as a timeinvariant approach). Considering the idea of this approach, at the same time that time invariance is preserved, the wrapping effect is avoided due to the fact that uncertainty is not propagated from step to step but from the initial state. This approach yields the accurate time-invariant worst-case observation without any conservatism, assuming that the previous optimization problems could be solved with infinite precision and the global optimum could be determined. However, in practice it only could be solved with a given precision. On the other hand, one of the main drawbacks of this approach, besides its high computational complexity, is that the objective function is a polynomial with degree increasing by one at each iteration. As a result, the amount of needed computation increases with time being impossible to operate over a large time interval. Moreover, according to Cugueró, Puig, Saludes, \& Escobet (2002), the wrapping effect affects to those interval models which are not monotonic since there are some elements of their model state-space matrix which are negative. Thus, when modelling a non-monotonic interval system using the interval observation approach, an monotonic interval observer (5) could be obtained designing the observer gain matrix $L$. Therefore, its state-space matrix $A_{0}$ becomes monotonic in spite of the non-monotonicity of system state-space matrix having in mind that based on Chen \& Patton (2012), the time-invariant uncertainty $\theta \in \Theta$ and using Lemma 1 and Assumption 2.1, the following inequality is stated:

$$
\underline{\xi_{k}} \leq \Delta A(\theta) x_{k} \leq \overline{\xi_{k}} .
$$

Moreover, considering the functions for upper and lower bounds based on the relationship between the state and parameters from initial state to the current state, the effect of initial state can be neglected in (42). Thus, when designing the observer to result in a monotonic system, the solution of (42) is achieved using the extreme values of the bounded uncertainties that can be formulated as in (39). Then, (39) can guarantee the iterative solution of the optimization problem in (42), the numerical solution of (38) considering Lemma 1. In this regard, the loss of the time dependency of the parametric uncertainty in the set-based interval observer approach and the problem of wrapping effect can also be avoided.

Remark 4.2. In fact, the main goal of using Lemma 1 is to guarantee that $\underline{x} \leq$ $x \leq \bar{x}$. But, since bounded uncertainties are considered for the case of time-varying uncertainties, it is already guaranteed that $\underline{E}_{d} d_{k}<E_{d} d_{k}<\overline{E_{d} d_{k}}$, where $\overline{E_{d}}$ and $\underline{E_{d}}$ are the constant monotonic matrices, and $\overline{\overline{d_{k}}}$ and $d_{k}$ are the extreme values of the time-varying bounded uncertainties.

Consequently, the estimation of the lower and upper bounds of the output measurement $y$ can be computed as

$$
\begin{aligned}
& \overline{y_{k}}=C^{+} \overline{x_{k}}-C^{-} \underline{x_{k}}, \\
& \underline{y_{k}}=C^{+} \underline{x_{k}}-C^{-} \overline{x_{k}},
\end{aligned}
$$

where $C^{+}=\max \{0, C\}$ and $C^{-}=C^{+}-C$. 


\subsection{Robust interval observer design}

There are two issues that should be taken into account when designing the robust observer in the case of the proposed interval observer approach. First, the convergence of the observer should be guaranteed, which will be done based on the $H_{\infty}$ technique. Second, the monotonicity of the observer in spite of non-monotonicity of the system state matrix should also be satisfied (see Property 5 in the Appendix). In this regard, both the Lyapunov stability condition and the following condition should be fulfilled together:

$$
\left(A_{0}-\bar{L} C\right),\left(A_{0}-\underline{L} C\right)>0 \text {, or, }\left(A_{0}-\bar{L} C\right),\left(A_{0}-\underline{L} C\right) \in \mathbb{R}_{+}^{n_{x} \times n_{x}} .
$$

The dynamics of state estimation error can be obtained by following Proposition 4.2, which are required for using the $H_{\infty}$ technique.

Proposition 4.2. Given that the upper and lower bounds of the state estimation error for the proposed interval observer approach are respectively defined as

$$
\begin{aligned}
& \overline{e_{k+1}}=\overline{x_{k+1}}-x_{k+1}, \\
& \underline{e_{k+1}}=x_{k+1}-\underline{x_{k+1}},
\end{aligned}
$$

then, based on (1) and (39), and also considering Lemma 2, the upper and lower bounds dynamics of the state estimation error can be obtained as

$$
\begin{aligned}
& \overline{e_{k+1}}=\left(A_{0}-\bar{L} C\right) \overline{e_{k}}+\overline{E_{e_{d}}} \overline{e_{d_{k}}}, \\
& \underline{e_{k+1}}=\left(A_{0}-\underline{L} C\right) \underline{e_{k}}+\underline{E_{e_{d}}} \underline{\underline{e_{d_{k}}}},
\end{aligned}
$$

where

$$
\begin{array}{ll}
\overline{E_{e_{d}}}=\left[\begin{array}{ll}
\overline{E_{d}} & E_{\theta}
\end{array}\right], & \overline{e_{d_{k}}}=\left[\begin{array}{ll}
\overline{d_{k}} & \overline{e_{\theta}}
\end{array}\right]^{\top}, \\
\underline{E_{e_{d}}}=\left[\begin{array}{ll}
\underline{E_{d}} & E_{\theta}
\end{array}\right], & \underline{e_{d_{k}}}=\left[\begin{array}{ll}
\underline{d_{k}} & \underline{e_{\theta}}
\end{array}\right]^{\top},
\end{array}
$$

where $\overline{e_{\theta}}$ and $e_{\theta}$ show the effect of the time-invariant uncertain parameter $\theta$ on the upper-and lower-bound dynamics of the state estimation error, respectively, i.e.,

$$
\begin{aligned}
& \overline{e_{\theta}}=\bar{\theta}-\theta, \\
& \underline{e_{\theta}}=\underline{\theta}-\theta .
\end{aligned}
$$

Proof. By substituting (1) and (39) in (46a), it can be written that

$$
\begin{aligned}
\overline{e_{k+1}}= & \left(A_{0}-\bar{L} C\right) \overline{x_{k}}-\left(A_{0}-\bar{L} C\right) x_{k}+\bar{L} E_{v} v_{k}+E_{\omega} \overline{\omega_{k}} \\
& -\bar{L} E_{v} \overline{v_{k}}+\overline{\xi_{k}}-\Delta A(\theta) x_{k}-E_{\omega} \omega_{k} .
\end{aligned}
$$


Then, (49) can be rearranged as

$$
\begin{aligned}
\overline{e_{k+1}}= & \left(\left(A_{0}-\bar{L} C\right)\left(\overline{x_{k}}-x_{k}\right)\right)+\left(E_{\omega}\left(\overline{\omega_{k}}-\omega_{k}\right)\right) \\
& -\left(\bar{L} E_{v}\left(\overline{v_{k}}-v_{k}\right)\right)+\left(\overline{\xi_{k}}-\Delta A\left(\theta_{k}\right) x_{k}\right)
\end{aligned}
$$

Based on (43) and introducing the following parameters:

$$
\overline{e_{\omega_{k}}}=\overline{\omega_{k}}-\omega_{k}, \quad \overline{e_{v_{k}}}=\overline{v_{k}}-v_{k}, \quad \overline{e_{\xi_{k}}}=\overline{\xi_{k}}-\Delta A\left(\theta_{k}\right) x_{k},
$$

then $(50)$ can be rewritten as

$$
\overline{e_{k+1}}=\left(\left(A_{0}-\bar{L} C\right) \overline{e_{k}}\right)+\left(E_{\omega} \overline{e_{\omega_{k}}}\right)-\left(\bar{L} E_{v} \overline{e_{v_{k}}}\right)+\left(\overline{e_{\xi_{k}}}\right) .
$$

Moreover, considering Lemma 2, the term $\overline{e_{\xi_{k}}}$ can be approximately computed as

$$
\overline{e_{\xi_{k}}} \approx E_{\theta} \overline{e_{\theta}} .
$$

Hence, considering (51), the upper bound dynamics of the state estimation error can be derived as

$$
\overline{e_{k+1}}=\left(\left(A_{0}-\bar{L} C\right) \overline{e_{k}}\right)+\left(E_{\omega} \overline{e_{\omega_{k}}}\right)-\left(\bar{L} E_{v} \overline{e_{v_{k}}}\right)+\left(E_{\theta} \overline{e_{\theta}}\right) .
$$

Therefore, the upper bound dynamics of the state estimation error in (47a) can be obtained by substitution of the terms in (48a) and (40a) into (52). Following the same procedure, $(47 \mathrm{~b})$ can be obtained for the lower bound dynamics of the state estimation error.

Now, by defining the transfer function $\mathscr{G}_{e d}(z)$ for the upper and lower state estimation error dynamics as $\overline{\mathscr{G}_{e d}(z)}$ and $\mathscr{G}_{e d}(z)$, the maximum singular value $\left(H_{\infty}\right.$ norm) of

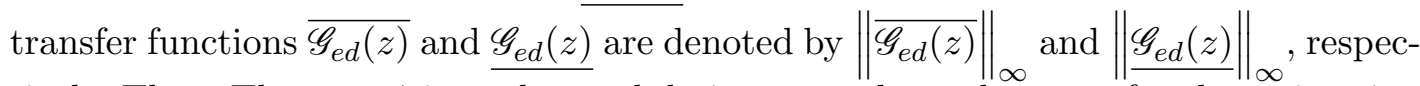
tively. Then, Theorem 4.3 can be used design two robust observers for the estimation of the upper and lower bounds of state-bounding observer considering the effect of uncertainties. Thus, both convergence and monotonicity properties of the observer are considered to design such observers.

Theorem 4.3. Taking into account the satisfaction of the monotonicity property and given a scalar $\gamma>0$, upper and lower state estimation error dynamics in (47) are stable and satisfy the following $H_{\infty}$ performance indices:

$$
\left\|\overline{\mathscr{G}_{e d}(z)}\right\|_{\infty}<\gamma, \quad\left\|\underline{\underline{\mathscr{G}_{e d}(z)}}\right\|_{\infty}<\gamma
$$

if there exists a symmetric positive definite matrix $\bar{P} \in \mathbb{R}^{n_{x} \times n_{x}}$ and a matrix $\bar{M} \in$ 
$\mathbb{R}^{n_{x} \times n_{y}}$ such that

$$
\begin{gathered}
{\left[\begin{array}{cccccc}
-\bar{P} & \bar{P} A_{0}-\bar{M} C & \bar{P} E_{\theta} & \bar{P} E_{\omega} & -\bar{M} E_{v} & 0 \\
* & -\bar{P} & 0 & 0 & 0 & I \\
* & * & \gamma I & 0 & 0 & 0 \\
* & * & * & \gamma I & 0 & 0 \\
* & * & * & * & \gamma I & 0 \\
* & * & * & * & * & \gamma I
\end{array}\right]<0,} \\
\bar{P} A_{0}-\bar{M} C \geq 0 .
\end{gathered}
$$

Analogously, for the lower observer, if there exists a symmetric positive definite matrix $\underline{P} \in \mathbb{R}^{n_{x} \times n_{x}}$ and a matrix $\underline{M} \in \mathbb{R}^{n_{x} \times n_{y}}$ such that

$$
\begin{aligned}
& {\left[\begin{array}{cccccc}
-\underline{P} & \underline{P} A_{0}-\underline{M} C & \underline{P} E_{\theta} & \underline{P} E_{\omega} & -\underline{M} E_{v} & 0 \\
* & -\underline{P} & 0 & 0 & 0 & I \\
* & * & \gamma I & 0 & 0 & 0 \\
* & * & * & \gamma I & 0 & 0 \\
* & * & * & * & \gamma I & 0 \\
* & * & * & * & * & \gamma I
\end{array}\right]<0,} \\
& \underline{P} A_{0}-\underline{M C} \geq 0,
\end{aligned}
$$

thus, solving the LMIs in (54) and (55), the gain of upper observer $\bar{L}$ and lower observer $\underline{L}$ can be respectively obtained as

$$
\begin{aligned}
& \bar{L}=\bar{P}^{-1} \bar{M}, \\
& \underline{L}=\underline{P}^{-1} \underline{M} .
\end{aligned}
$$

Proof. The proof follows following the same procedure as it the one used to proof Theorem 3.4. They only differ from the second LMI for upper and lower observers to satisfy the monotonicity property, i.e., $A_{0}-L C>0$.

Algorithm 2 summarizes the state estimation using the proposed interval observer approach.

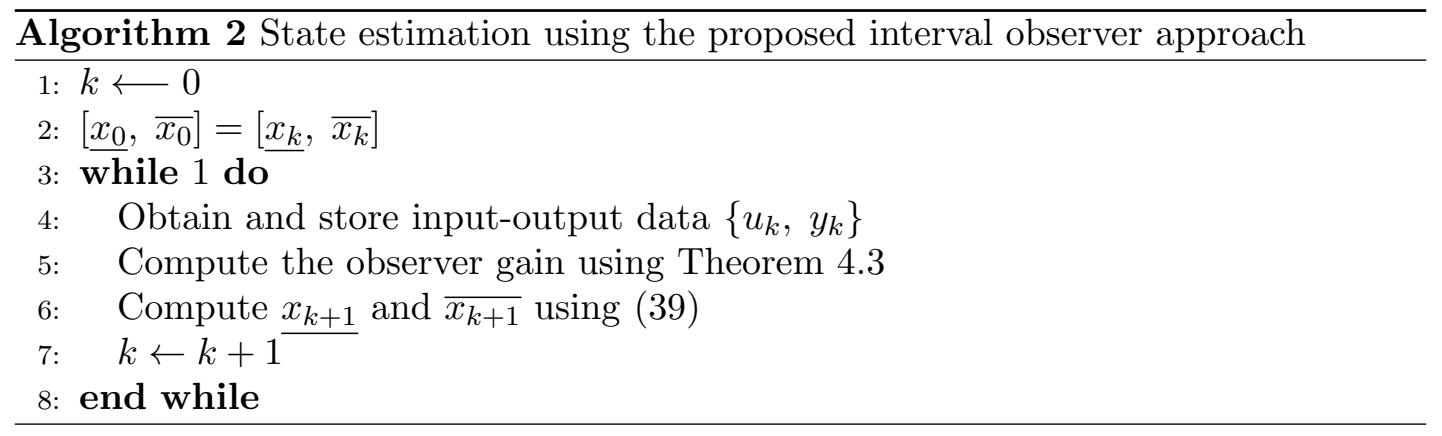

\section{Integrated approach}




\subsection{Comparative assessment}

Using the results presented in Sections 3 and 4, the state-bounding observer can be designed by using both (12) and (39), which corresponds, respectively, to the setbased and proposed interval observer approaches. According to Section 3.2, in the set-based interval observer approach, the satisfaction of the LMI in Theorem 3.4 is required in order to guarantee the robustness of the interval observation. Moreover, the size of the obtained state-bounding zonotope can be minimized using the LMI (19) in Theorem 3.5. On the other hand, according to Section 4.2, both robustness and monotonicity property of the proposed interval observer approach can be guaranteed through the satisfaction of the LMIs (54) and (55) in Theorem 4.3 for computing the gains of the upper and lower observers.

In an attempt to make both approaches comparable, the interval hull ${ }^{2}$ introduced in Definition 5 in the Appendix is used for the case of set-based interval observer approach. In this regard, the interval hull of the state-bounding zonotopic set in (12) can be written as

$$
\begin{aligned}
x_{k, \text { sup }, i} & =c_{k, i}+\left\|R_{k, i}\right\|_{1}, \\
x_{k, \text { inf }, i} & =c_{k, i}-\left\|R_{k, i}\right\|_{1},
\end{aligned}
$$

where $x_{k, \text { sup }} \in \mathbb{R}^{n_{x}}$ and $x_{k, \text { inf }} \in \mathbb{R}^{n_{x}}$ are the maximum and the minimum values of the $\mathcal{X}$, respectively. Moreover, $\left\|R_{k, i}\right\|_{1}$ stands for row sum where $R_{k, i}$ is the $i$-th row of $R_{k}$. In Figure 1, there is a schematic diagram of the interval hull for a two-dimensional zonotope.

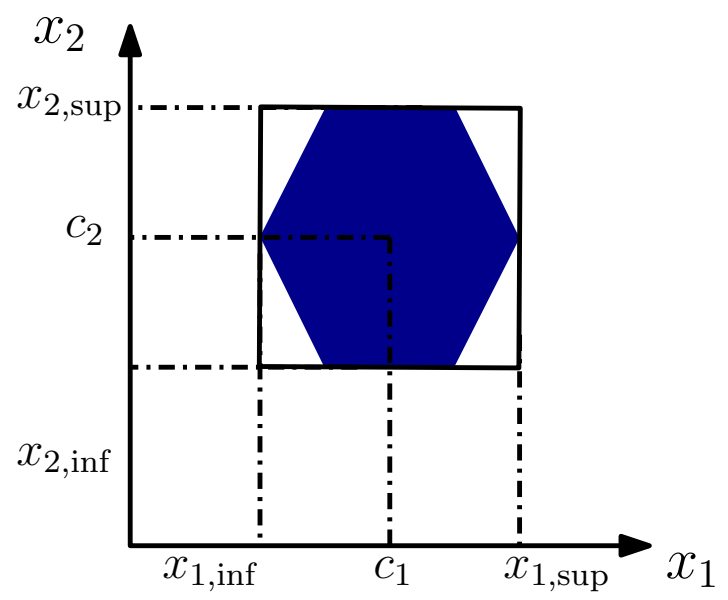

Figure 1. Schematic diagram of the interval hull of a two-dimensional zonotope.

Therefore, considering (57) instead of (12) leads to compute the extreme values of the trajectories of the state-bounding set in the set-based interval observer approach. Since the main structure of the proposed interval observer approach relies on computing the extreme values of the state estimation, using the concept of interval hull for the set-based interval observer approach allows to compare the results of the set-based and proposed interval observer approaches.

By looking at both approaches with the purpose of further analysis, Proposition 5.1 can be used in order to compare the nominal values of the state estimation that can

\footnotetext{
${ }^{2} \mathrm{An}$ interval hull of a set is defined as the smallest centered interval vector that contains the set.
} 
be obtained using each approach.

Proposition 5.1. Considering an interval observer with symmetric uncertainty satisfying the monotonicity property, the mean value of computed upper and lower bounds of the state estimation using (39) with $\bar{L}=L=L$, the mean value in the case of the proposed interval observer approach plays the same role as the center of the zonotope in the set-based observer approach, i.e.,

$$
c_{k, i}=\frac{\overline{x_{k, i}}+\underline{x_{k, i}}}{2}
$$

where the index $i$ refers to the rows of the vector.

Proof. Let consider a interval observer designed to satisfy the monotonic property observer and assuming $\bar{L}=\underline{L}=L$. Moreover, assuming that the extreme values of uncertainties (for the upper and lower observers) are equal, i.e., $\overline{d_{k}}=\underline{d_{k}}$ and $\overline{\xi_{k}}=\underline{\xi_{k}}$, the mean value of $\overline{x_{k+1}}$ and $x_{k+1}$ can be obtained using (39) as

$$
\begin{aligned}
\left(\frac{\overline{x_{k+1, i}}+\underline{x_{k+1, i}}}{2}\right)= & \left(A_{0}-L C\right)\left(\frac{\overline{x_{k, i}}+\underline{x_{k, i}}}{2}\right)+B u_{k, i}+L y_{k, i} \\
& +E_{d}\left(\frac{\overline{d_{k, i}}+\underline{d_{k, i}}}{2}\right)+\left(\frac{\overline{\xi_{k, i}}+\underline{\xi_{k, i}}}{2}\right) .
\end{aligned}
$$

Now, by considering Assumption 3.1, the mean value of upper and lower bounds of the state estimation using (39) can be written as

$$
\left(\frac{\overline{x_{k+1, i}}+\underline{x_{k+1, i}}}{2}\right)=\left(A_{0}-L C\right)\left(\frac{\overline{x_{k, i}}+\underline{x_{k, i}}}{2}\right)+B u_{k, i}+L y_{k, i} .
$$

Then, comparing the right-hand side of (60) and (12a), it can be seen that the center of the state-bounding observer using the set-based interval observer approach and the mean values of both $\overline{x_{k}}$ and $\underline{x_{k}}$ plays the same role.

Remark 5.1. It is worth mentioning that the equality in (58) is satisfied when the observer is monotonic with symmetric uncertainty. Otherwise, there exists over approximation and the condition in (58) is not longer satisfied.

On the other hand, the width between the upper and lower bounds ${ }^{3}$ that are computed using both set-based and proposed approaches for the state estimation is related in Proposition 5.2.

Proposition 5.2. Given the interval hull in Definition 5, the relationship between the shape matrix considering the set-based interval observer approach and the extreme values of the interval observation provided by the proposed interval observer approach can be written as

$$
\begin{aligned}
x_{k, \sup , i} & =c_{k, i}+\left\|R_{k, i}\right\|_{1}=\overline{x_{k, i}}, \\
x_{k, \inf , i} & =c_{k, i}-\left\|R_{k, i}\right\|_{1}=\underline{x_{k, i}} .
\end{aligned}
$$

\footnotetext{
${ }^{3}$ This part shows the effect of the uncertainties since the deterministic approach is used.
} 
Proof. Based on Definition 5, the interval hull of the state bounding observer can be obtained as (57). Then, having in mind that the interval computed from the upper and lower observers in the proposed interval observer approach plays the same role as the interval hull computed using the set-based interval observer approach, it can be written that

$$
\begin{aligned}
x_{k, \text { sup }, i} & =\overline{x_{k, i}}, \\
x_{k, \text { inf }, i} & =\underline{x_{k, i}} .
\end{aligned}
$$

Then, considering (57), (61) can be obtained from (62).

\subsection{Integrated scheme}

By considering the relationship between both interval observer approaches, an integrated scheme combining them is proposed for interval observation. This integrated approach can be obtained using Propositions 5.1 and 5.2 as it is proposed in Theorem 5.3.

Theorem 5.3. Given Propositions 5.1 and 5.2, the nominal value of the statebounding observer $x_{\text {nom }}$ in the case of the proposed interval observer approach is equal to the center of the zonotope in the case of set-based interval observer approach. Then, it can be written that

$$
x_{k+1, \mathrm{nom}}=\left(A_{0}-L C\right) x_{k, \mathrm{nom}}+B u_{k}+L y_{k} .
$$

Moreover, having in mind the same idea as Proposition 4.1, given the interval hull in Definition 5 and considering (61), the effect of both considered time-varying and time-invariant uncertainties (by using Lemma 2) can be bounded as

$$
\begin{aligned}
x_{k+1, \text { sup }} & =\left(A_{0}-L C\right) x_{k, \text { sup }}+B u_{k}+L y_{k}+\overline{E_{d} d_{k}}+\overline{\xi_{k}}, \\
x_{k+1, \mathrm{inf}} & =\left(A_{0}-L C\right) x_{k, \mathrm{inf}}+B u_{k}+L y_{k}+\underline{E_{d} d_{k}}+\underline{\xi_{k}},
\end{aligned}
$$

where the gain $L=P^{-1} M$ should be computed using the same LMI in (19) to satisfy the $H_{\infty}$ performance together with the new LMI

$$
P A_{0}-M C \geq 0,
$$

to satisfy the monotonicity property of $\left(A_{0}-L C\right)$.

Proof. Given Proposition 5.1 and assuming that $\bar{L}=\underline{L}=L$, the nominal value of the state estimation can be obtained using the computed center in (60) as (63). Moreover, using Proposition 5.2, the effect of the uncertainties can be alternatively computed as (64).

Moreover, the proof of the mentioned LMIs in (19) and (65) to compute the observer gain can be obtained following the same manner as Theorems 3.4 and 4.3 to satisfy both $H_{\infty}$ performance and monotonicity property, respectively.

Theorem 5.3 shows that both approaches can be merged, generating a new approach where the center and segments of the state-bounding observer are propagated independently (as in zonotopic approach), but obtaining explicit formulas that do not require 
the use of zonotopes. However, since the observer structure in the set-based interval observer approach is reformulated using the interval hull of the state-bounding zonotope for computing the upper and lower bounds as in the proposed interval observer approach, the integrated scheme will only avoid the wrapping effect and preserve the parameter uncertainty time invariance if the observer gain is designed such that the resulting observer matrix satisfies the monotonicity property. Otherwise, the integrated scheme will not work satisfactorily leading to an unstable interval observer due to the wrapping effect (Puig et al., 2005a). Moreover, the robustness of the observer can be guaranteed considering the $H_{\infty}$ performance when computing the observer gain using Theorem 3.5.

Corollary 5.4. Using Theorem 5.3, the set-based and proposed interval observer approaches can produce the same results when the observer is monotonic.

Proof. Given a system dynamic (10) and using the structure of the proposed interval observer approach in Section 4, the extreme values of upper and lower bounds are computed as

$$
\begin{aligned}
& \overline{x_{k+1}}=\left(A_{0}-\bar{L} C\right) \overline{x_{k}}+B u_{k}+\bar{L} y_{k}+\overline{E_{d}} \overline{d_{k}}+E_{\theta} \bar{\theta}_{k}, \\
& \underline{x_{k+1}}=\left(A_{0}-\underline{L} C\right) \underline{x_{k}}+B u_{k}+\underline{L} y_{k}+\underline{E_{d}} \underline{d_{k}}+E_{\theta} \underline{\theta} .
\end{aligned}
$$

On the other hand, the main concept of using the set-based interval observer approach to compute the state-bounding observer is to generate both upper and lower bounds using some propagation algorithms (such as the algorithm mentioned in Proposition 3.1) to compute the set $\mathcal{X}_{k+1}$ from $\mathcal{X}_{k}$. Then, it can be written that

$$
\begin{aligned}
x_{k+1} \in\left\langle c_{k+1}, R_{k+1}\right\rangle= & \left\langle\left(A_{0}-L C\right) c_{k},\left(A_{0}-L C\right) \check{R}_{k}\right\rangle \oplus\left\langle B u_{k}, 0\right\rangle \\
& \oplus\left\langle L y_{k}, 0\right\rangle \oplus\left\langle 0, E_{w}\right\rangle \oplus\left\langle 0,-L E_{v}\right\rangle \oplus\left\langle 0, E_{\theta}\right\rangle .
\end{aligned}
$$

Now, given the relationship obtained in Theorem 5.3, (67) can be reformulated using the concept of the interval hull to compute the same result as in (66).

Algorithm 3 summarizes the state estimation using the proposed integrated interval observer approach.

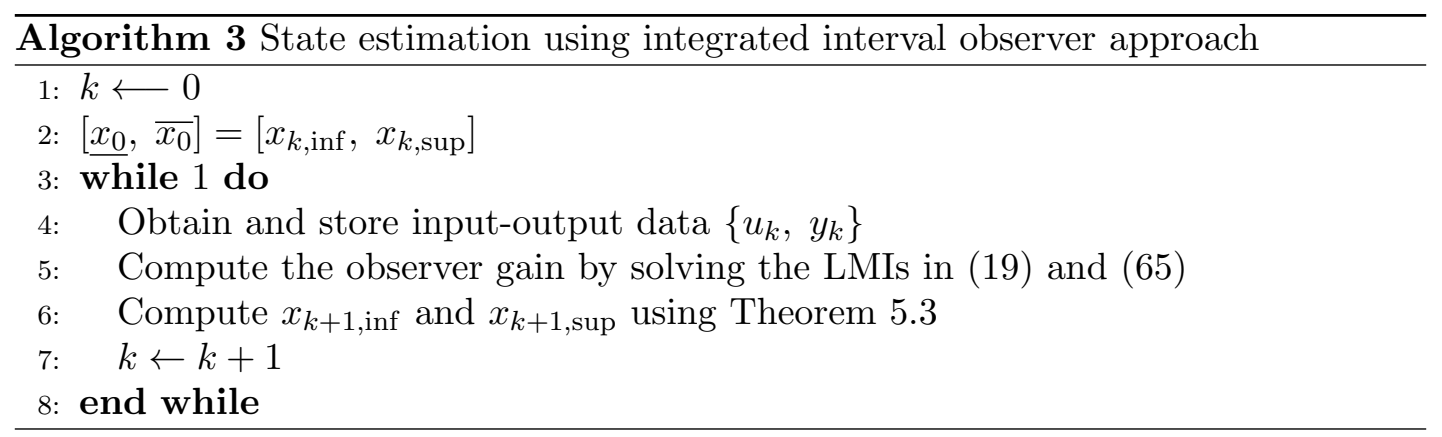

\section{Case study}

Two examples are used for illustrating the effectiveness of the approaches proposed in the previous sections: a numerical example and a real application example (the well- 
known two-tanks system benchmark). Generally speaking, these application examples are selected to show their performance: i) when the system is non-monotonic, i.e., at least one element of system matrix $A$ is negative, ii) when the observer is nonmonotonic, i.e., at least one element of observer matrix $\left(A_{0}-L C\right)$ is negative. In both cases, the proposed observer design is used to overcome the problems using both set-based and proposed interval observer approaches.

\subsection{Numerical example}

The first example considered is based on the dynamical model (1) with

$$
A=\left[\begin{array}{ccc}
0.8+\theta_{11} & 0.1+\theta_{12} & 0.3+\theta_{13} \\
0+\theta_{21} & 0.8+\theta_{22} & 0.2+\theta_{23} \\
0.01+\theta_{31} & 0+\theta_{32} & 0.8+\theta_{33}
\end{array}\right], B=\left[\begin{array}{l}
0 \\
0 \\
1
\end{array}\right], C=\left[\begin{array}{lll}
0 & 0 & 1
\end{array}\right],
$$

where the time-invariant uncertain parameters are bounded by the interval $\theta_{i j} \in$ $[-0.01,0.01]$, where the indices $i$ and $j$ refer to the $i^{\text {th }}$ line and $j^{\text {th }}$ column of $\Delta A(\theta)$, respectively. Moreover, the example includes time-varying uncertainty, i.e., the state disturbance and the measurement noise, $E_{\omega}$ and $E_{v}$ are used as in (1) with

$$
E_{\omega}=\left[\begin{array}{ccc}
0.08 & 0 & 0 \\
0 & 0.08 & 0 \\
0 & 0 & 0.08
\end{array}\right], \quad E_{v}=[0.2]
$$

The input signal $u$ is given by $u=\sin (t)$ for $t \in[0,3 \pi]$ with 200 time steps.

Considering the results presented in the previous sections, it is worth comparing the proposed interval observer approach in Section 4 and set-based interval observer approach for the case of a monotonic system. In this regard, matrix $A$ is considered as in (68). It can be seen that all the entries of $A$ in (68) are positive values. Therefore, the system is a monotonic system. Then, the set-based interval observer approach gain is computed using Theorem 3.4 to satisfy the $H_{\infty}$ performance together with Theorem 3.5 to minimize the size of the obtained zonotope at each time instant. Then, it yields $L=\left[\begin{array}{lll}0.2781 & 0.2855 & 0.7982\end{array}\right]^{\top}$. Furthermore, the gains of upper and lower bounds of the proposed interval observer approach in Section 4 are computed using (54) and (55), respectively. In this case, it yields $\bar{L}=\underline{L}=\left[\begin{array}{lll}0.3 & 0.2 & 0.8\end{array}\right]^{\top}$. Figure 2 shows the obtained results from the simulation of the monotonic system.

Remark 6.1. It is worth mentioning that the set-based approach allows to estimate the state set one time instant ahead based on the set estimated in the previous time instant. But, in order to put both approaches into the comparable framework, the state sets are projected into the state space separately.

As it can be seen from Figure 2, the behavior of the system can be correctly estimated using both set-based and proposed interval observer approaches. Moreover, both mentioned approaches are producing the same results for the case of a monotonic system.

The problem appears when the considered system is not monotonic. To illustrate this situation, the scenario to be tested is the case when matrix $A$ contains at least 

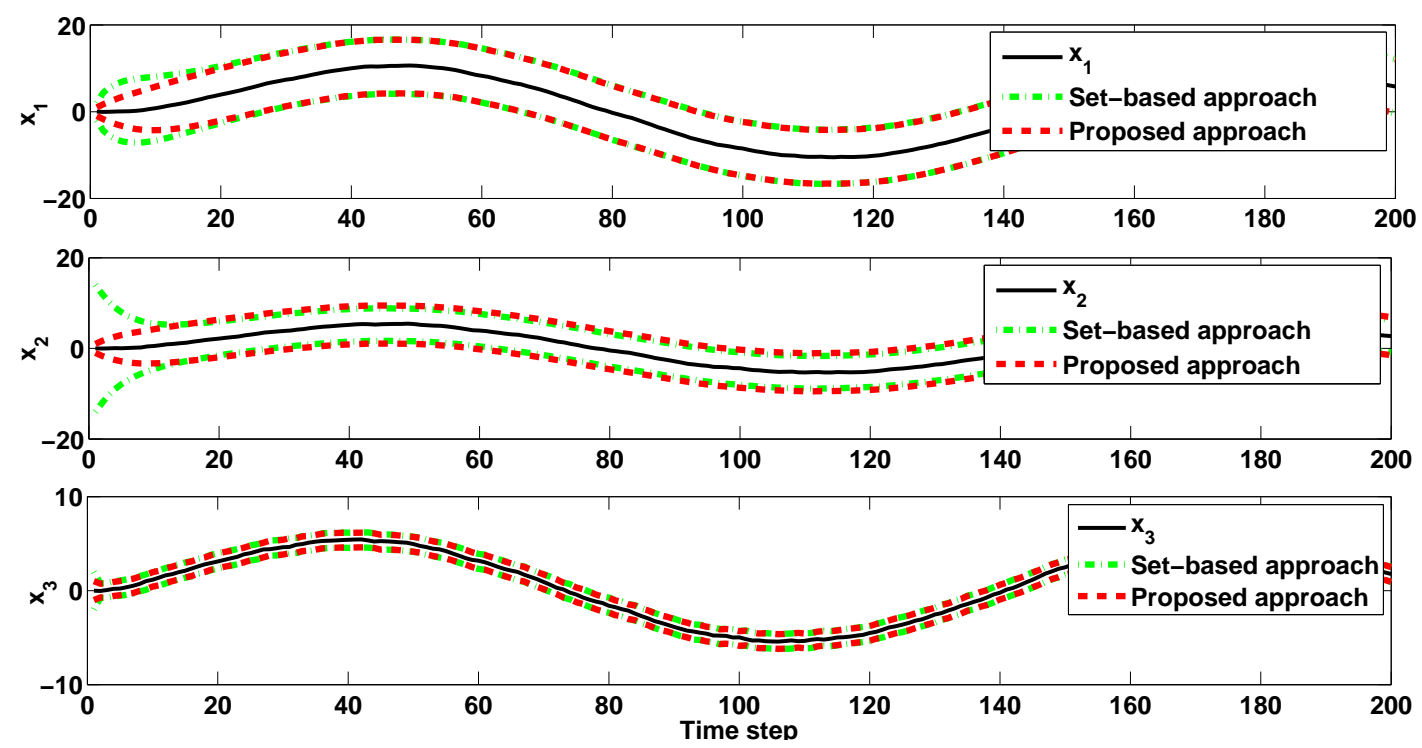

Figure 2. Set-based vs. proposed interval observer approach behavior considering monotonic system.

one negative element. To this aim, $A$ is now considered as

$$
A=\left[\begin{array}{ccc}
0.8+\theta_{11} & -0.1+\theta_{12} & 0.3+\theta_{13} \\
0+\theta_{21} & 0.8+\theta_{22} & 0.2+\theta_{23} \\
0.01+\theta_{31} & 0+\theta_{32} & 0.8+\theta_{33}
\end{array}\right]
$$

Having a negative element in matrix $A$ leads to test the case that the system is not monotonic since the positivity condition is not satisfied. The first simulation is the analysis of the behavior of the set-based and proposed interval observer approaches when only the stability condition is considered for designing the observer through the obtained LMI in (19) for the set-based interval observer approach, and (54a) and (55a) for the trajectory-based interval observer approach considering the $H_{\infty}$ technique. Figures 3 and 4 show the results obtained from the simulation of the non-monotonic system considering set-based and proposed interval observer approaches, respectively.

In Figure 3, the zonotopic observer is implemented according to Proposition 3.1 and the observer gain is determined using the LMI in (19) for the case of set-based interval observer approach. Then, it yields $L=\left[\begin{array}{lll}-1.1376 & -1.9167 & -1.2604\end{array}\right]^{\top}$. It can be observed from Figure 3 that, when the system is non-monotonic, the results from the set-based interval observer approach are affected by the problem of wrapping effect and this approach cannot compute the correct state estimation.

On the other hand, in Figure 4, the observer in (39) is used for implementing the proposed interval observer approach and the observer gain is calculated using LMIs in (54a) and (55a), respectively. In this case, it yields $\bar{L}=\underline{L}=$ $\left[\begin{array}{lll}0.2754 & 1.0571 & 0.7724\end{array}\right]^{\top}$. The idea of considering only (54a) and (55a) in the case of trajectory-based interval observer approach is to show the problem that appears when the positivity condition is not satisfied for the observer in the case that the dynamics of the system are not monotonic.

As it can be seen in Figure 4, considering only the convergence of the observer to compute the observer gain for a non-monotonic system, the proposed interval observer approach meets a problem to compute the interval for the estimation of the second 

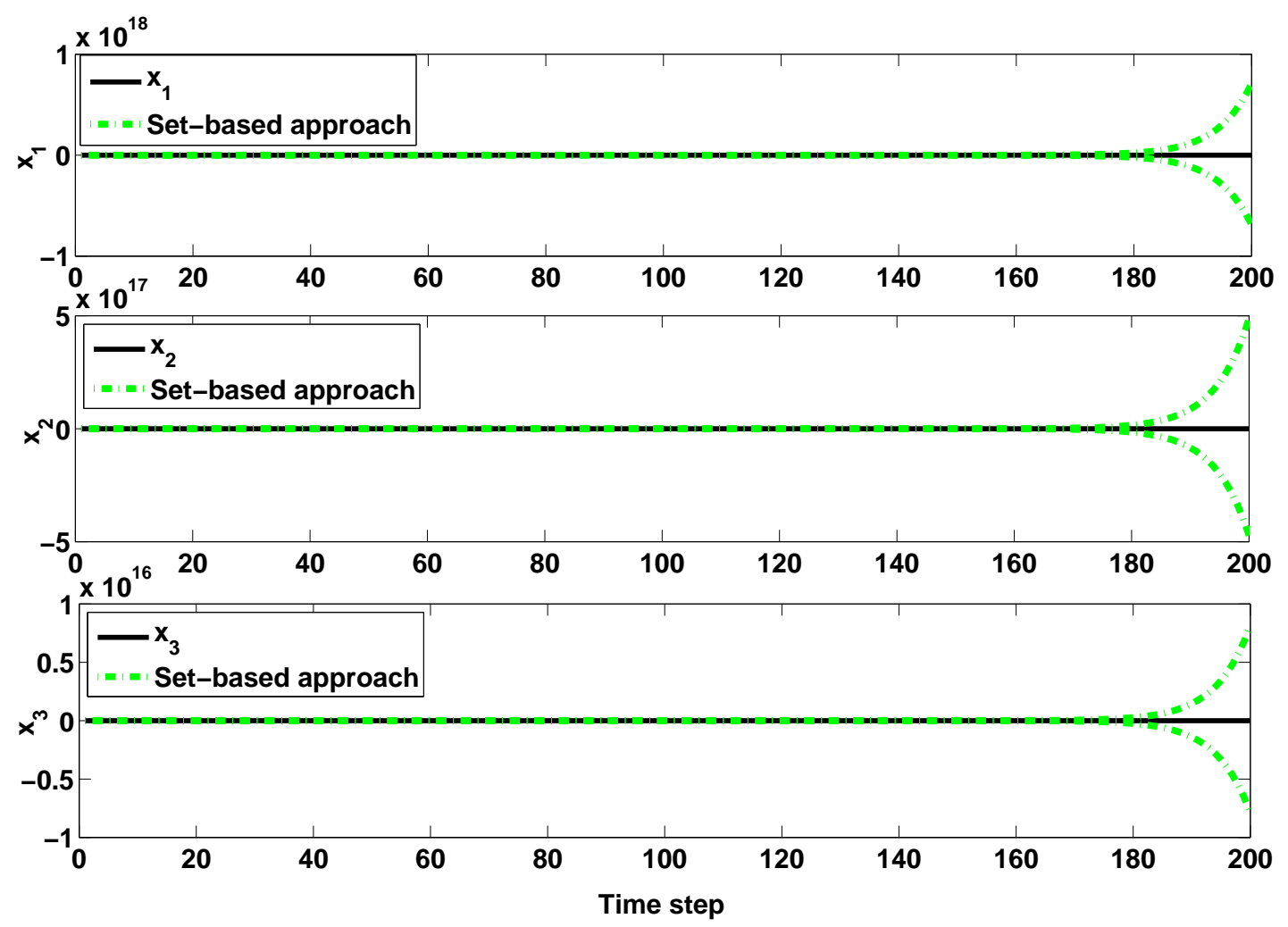

Figure 3. Set-based interval observer approach considering a non-monotonic system.

state of the system $x_{2}$ that, according matrix $C$, is not measured. The only purpose of considering this case is to show the problems of non-satisfaction of the positivity condition to design the observer gain in the proposed interval observer approach and having the wrapping effect for the same case considering the set-based interval observer approach.

Further analysis is done by implementing the proposed observer design in Section 5 to overcome the problems that are presented in Figures 3 and 4.

As it can be seen in Figure 5, the satisfaction of the positivity condition based on the proposed LMIs in (54) and (55) allows the proposed interval observer approach to estimate correctly the system behavior and to solve the problem of estimating the behaviour of $x_{2}$ (see Figure 4). Moreover, thanks to the obtained similarity between the set-based and proposed interval observer approaches in the case of a monotonic system according to the analysis of the numerical example and also considering the mentioned points in Section 5, it can be seen that the interval hull of the state-bounding zonotope computed using the set-based interval observer approach can be converted to the proposed interval observer approach. Having this in mind, Propositions 5.1 and 5.2, and Theorem 5.3 are considered for converting the center and the shape matrix of the state-bounding zonotope obtained from the set-based interval observer approach. This point is also shown in Figure 5.

As it is mentioned in Section 5 and having the integrated observer structure, the robust observer in Figure 5 is obtained by computing the observer gain considering the $H_{\infty}$ performance and the monotonicity property. It means, in the new proposed structure in Theorem 5.3, the observer gain is computed considering the LMIs in 


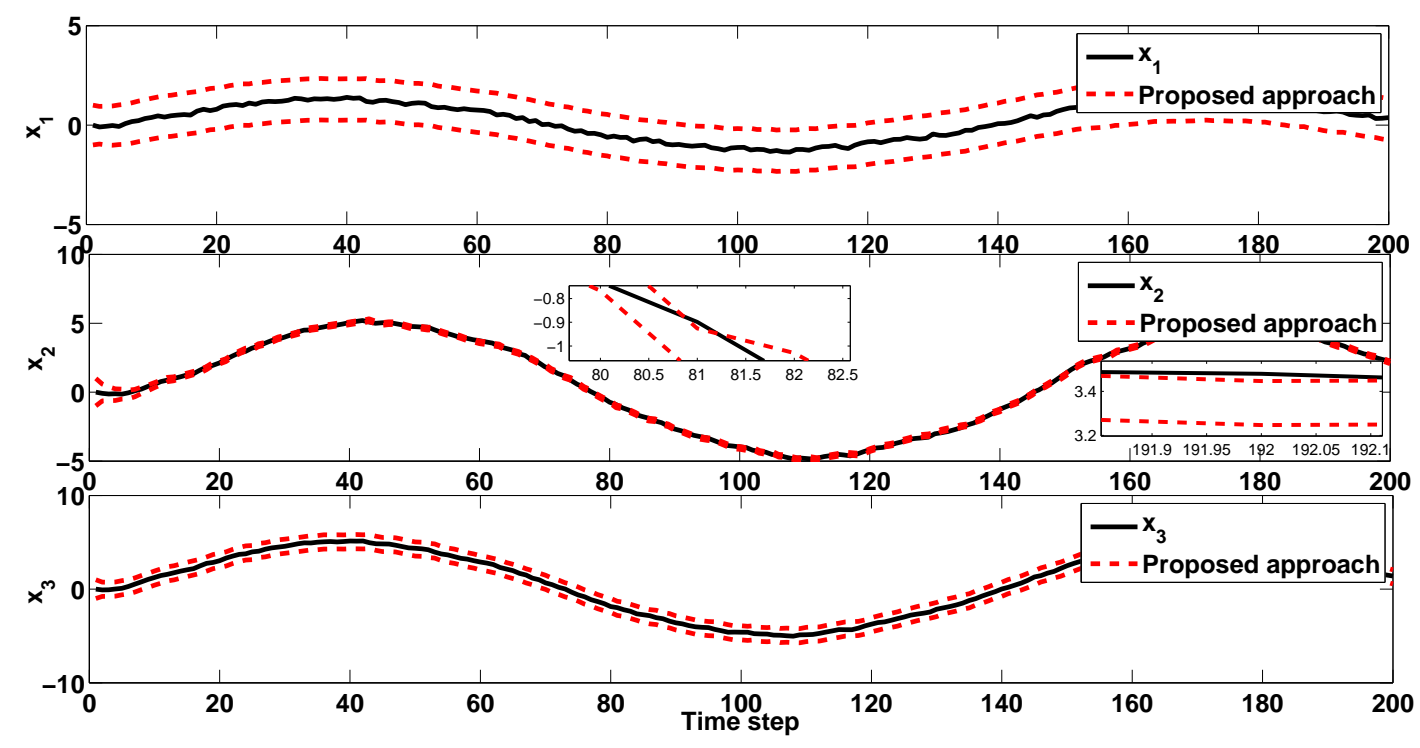

Figure 4. Proposed interval observer approach considering a non-monotonic system.

Theorem 3.4 and also the satisfaction of the LMI in (65), which guarantee the monotonicity property of the observer. Then, it yields $L=\left[\begin{array}{lll}0.2892 & 0.2950 & 0.8001\end{array}\right]^{\top}$. Note that both time-varying and time-invariant uncertainties are considered in the simulation, the monotonicity property not only can solve the non-inclusion problem of the trajectory-based interval observer approach but also the wrapping effect in the setbased interval observer can be solved. Furthermore, the same results are obtained from both approaches for the case that the system is non-monotonic and the observers are designed to satisfy the monotonicity property.

\subsection{Two-tank system benchmark}

A two-tank system is proposed as the second application example to illustrate the approach proposed in this paper. The considered tank process is based on Coupled Tanks 33 - 041, manufactured by Feedback Instruments company. The schematic diagram of the system setup of the considered case study is shown in Figure 6.

As it can be seen from the schematic diagram, the system contains a pump and two interconnected tanks. The main system input is the pump flow rate that is given by the voltage of the Pump 1. The action of pump is to fill the tanks by extracting the water from the basin. The input voltages to the pumps vary between $0 \mathrm{~V}$ to 5 $\mathrm{V}$, where $0 \mathrm{~V}$ means the pump is off and $5 \mathrm{~V}$ means the pump is working with its maximum power. Due to the gravity effect, Tank 1 is being affected by the outflow of Tank 2 due to the topology of the system. Both upper and lower tanks are made by Plexiglas tubes with a height of $25 \mathrm{~cm}$ and pipes with diameter $10 \mathrm{~mm}$ is utilized to make all the connections between the tanks and the pump. The output of the process is the water level in the lower tank that is obtained as a voltage from pressure sensor. Moreover, two valves are employed in order to control the water flow to each tank. As it can be seen from Figure 6, Valves 1 and 2 are used to control the inflow of the tanks.

The mathematical model of the process can be determined based on the mass bal- 

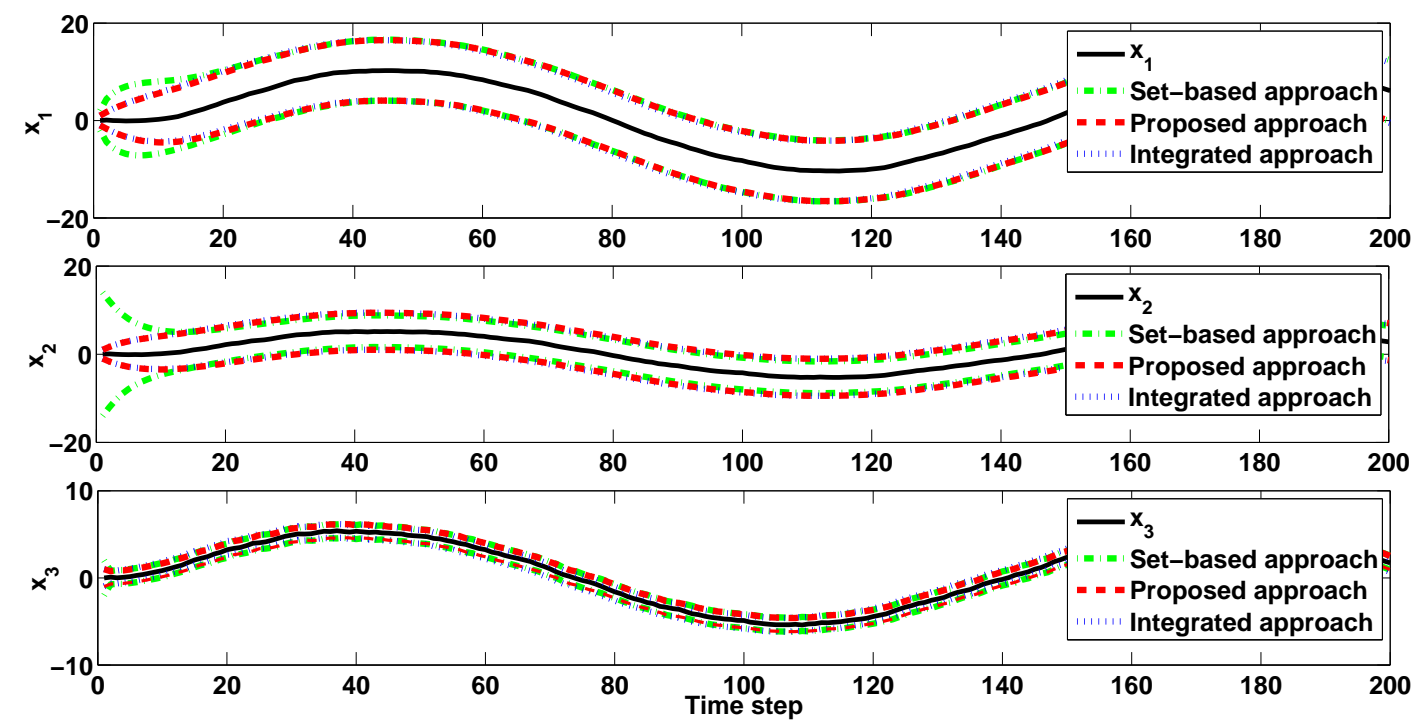

Figure 5. Set-based vs. proposed interval observer approaches considering non-monotonic system and designing the integrated monotonic observer.

ance relations and Bernoulli's law as

$$
\begin{aligned}
\frac{d h_{1, t}}{d t} & =-\frac{a_{1}}{A_{1}} \sqrt{2 g h_{1, t}}+\frac{a_{2}}{A_{1}} \sqrt{2 g h_{2, t}}+\frac{\gamma_{1} K_{p}}{A_{1}} u_{t}, \\
\frac{d h_{2, t}}{d t} & =-\frac{a_{2}}{A_{2}} \sqrt{2 g h_{2, t}}+\frac{\left(1-\gamma_{2}\right) K_{p}}{A_{2}} u_{t},
\end{aligned}
$$

where

- $\gamma_{i}$ is the valve $i$ ratio, with $i=1,2$;

- $K_{p} v_{t}$ is the flow through the pump;

- $\left(1-\gamma_{2}\right) K_{p} v_{t}$ is the flow towards Tank 2 according to the valve position $\gamma_{2}$;

- $\gamma_{1} K_{p} \omega_{t}$ is the flow towards Tank 1 according to the valve position $\gamma_{1}$;

- $K_{p}$ is the pump constant;

- $v_{t}$ is the velocity of the water flow through the pump;

- $A_{i}$ is the cross section of Tank $i$, with $i=1,2$;

- $a_{i}$ is the cross sectional area of the outlet pipes, with $i=1,2$;

- $g$ is acceleration due to gravity; and

- $h_{i}$ is the level of the water in Tank $i$, with $i=1,2$.

Furthermore, in order to design the process in such a way to be applicable with testing the proposed approach, the time-varying uncertainties, i,e., the state disturbance $\omega$ and process noise $v$ are generated placing a Pump 2 as can be seen in Figure 7 .

As it is shown in Figure 7, inflows of both tanks can also be affected by the additional disturbance $\omega$ and noise $v$ that are generated by the uncertain position of Valves 3 and 4, respectively. Moreover, the time-invariant uncertainty $\theta$ is implemented as an additional inflow using Pump 1. Hence, using the Euler discretization with a sampling time of $1 s$, a discrete-time linear model is obtained as in (1) with 


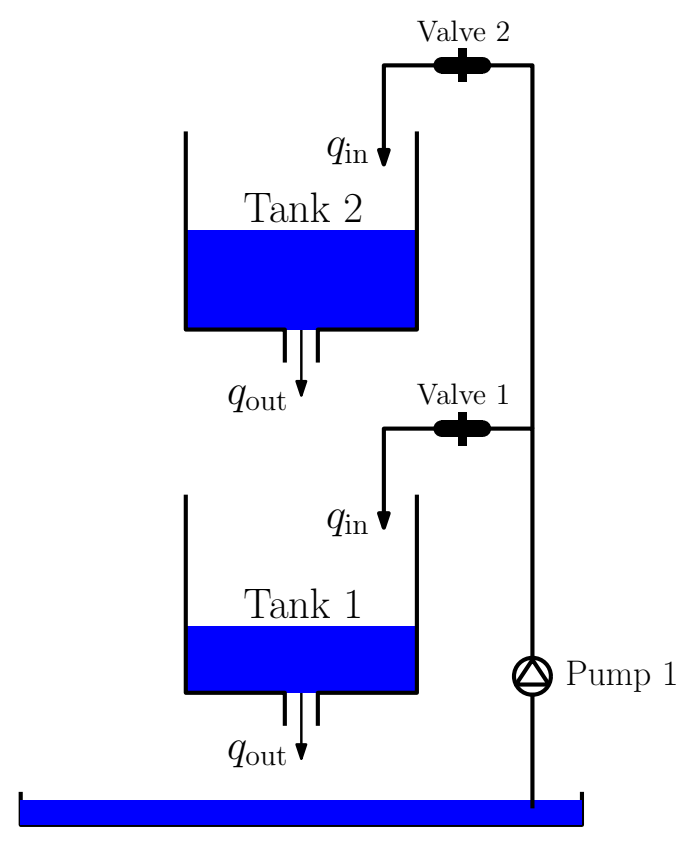

Figure 6. Schematic diagram of the tank system.

$$
\begin{aligned}
& A=\left[\begin{array}{lc}
0.9886+\theta_{11} & 0+\theta_{12} \\
0.0114+\theta_{21} & 0.9903+\theta_{22}
\end{array}\right], \quad B=\left[\begin{array}{c}
0.2261 \\
0
\end{array}\right], \\
& C=\left[\begin{array}{ll}
0 & 1
\end{array}\right]
\end{aligned}
$$

Moreover, the time-invariant uncertain parameters are bounded by the interval $\theta_{i j} \in$ $[-0.3 B,+0.3 B]$, and also, time-varying bounded disturbances influencing all the statespace directions and the measurement noise are modeled, respectively, with $E_{\omega}$ and $E_{v}$ as

$$
E_{\omega}=\left[\begin{array}{cc}
0.006 & 0 \\
0 & 0.006
\end{array}\right], \quad E_{v}=\left[\begin{array}{l}
0.5 \\
0.5
\end{array}\right]
$$

The input signal $u$ is given as it is shown in Figure 8 .

Two scenarios are considered in this section. Both are implemented using the same type of uncertainties. They differ from the observer structure used: set-based vs. proposed interval observer approaches as explained in previous sections. Furthermore, the zonotopic observer structure is considered based on Proposition 3.1 and the observer structure in (39) is used as a proposed interval observer approach structure.

As a first scenario, the computation of the observer gain is done using the LMI in (19) for the case of set-based interval observer approach yielding to $L=\left[\begin{array}{ll}0.6647 & 0.6542\end{array}\right]^{\top}$. Additionally, a proposed interval observer approach gain is calculated using LMIs in (54a) and (55a), respectively, leading to $\bar{L}=\underline{L}=$ $\left[\begin{array}{ll}0.6542 & 0.6493\end{array}\right]^{\top}$. The main purpose of having this analysis is to check the behavior of the state observation when only the stability condition is satisfied. Figure 9 shows the behavior of the set-based and proposed interval observer approaches tracking the height of the water in upper and lower tanks (obtained from the real system). 


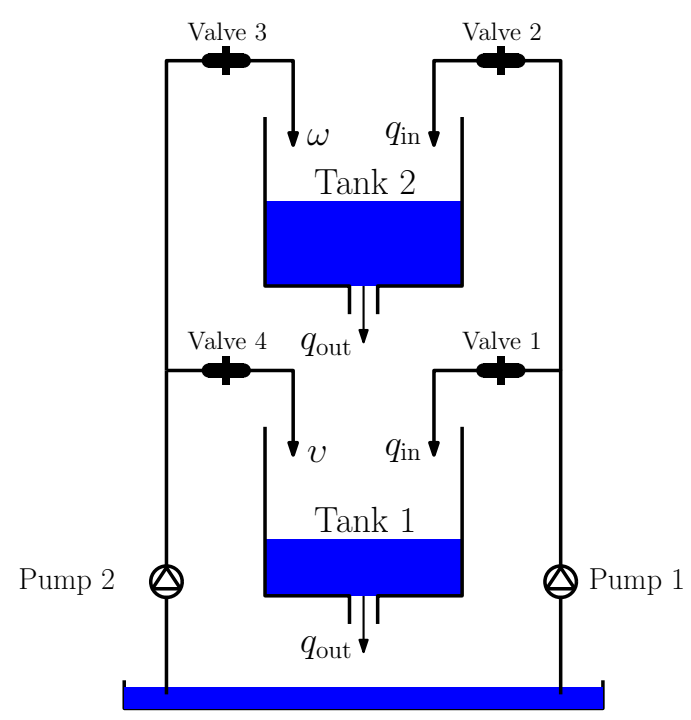

Figure 7. Schematic diagram of the tank system affected by the state disturbance and process noise.

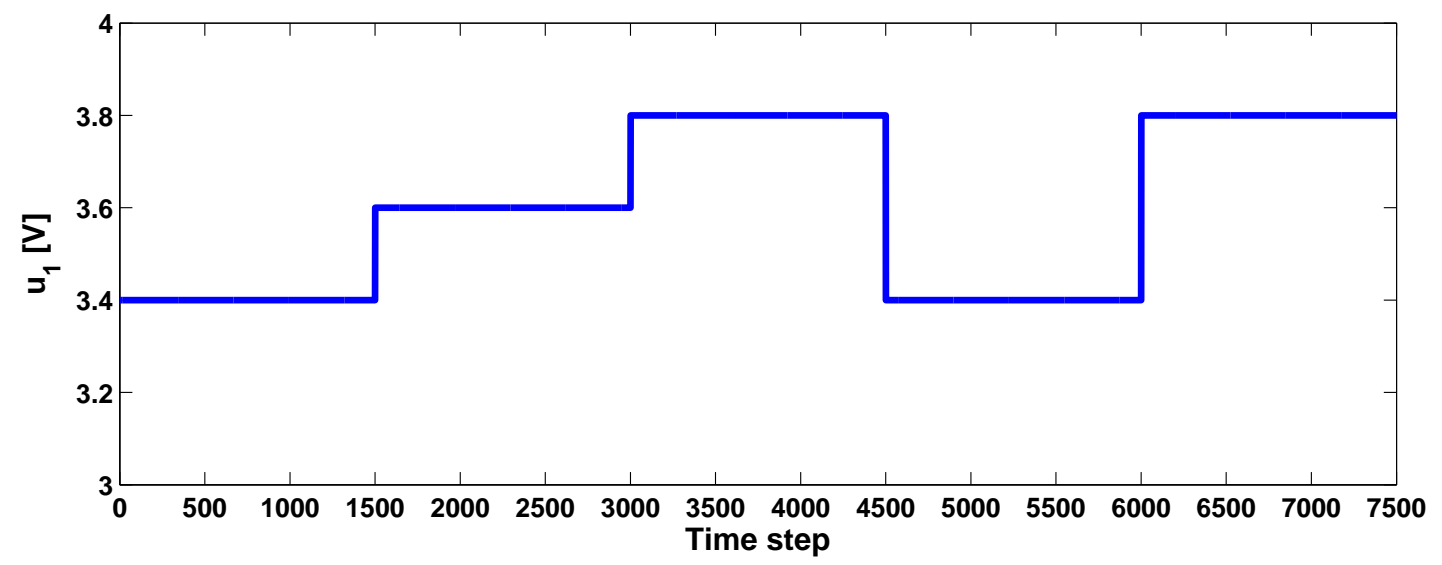

Figure 8. Pump signal.

As it can be seen from Figure 9, there is no problem in lower tank level estimation since it is measurable based on matrix $C$. But, using the trajectory-based interval observer approach, the correct estimation of the unmeasured state $h_{1}$ cannot be obtained. A possible explanation for this might be that the observer is not monotonic since the positivity condition is not considered for designing the observer gain. In order to solve this problem, the positivity condition is guaranteed by considering the LMIs in (54b) and (55b) for computing the observer gain together with (54a) and (55a) in trajectorybased interval observer approach. Then, it yields $\bar{L}=\underline{L}=\left[\begin{array}{ll}0.001 & 0.9803\end{array}\right]^{\top}$. Figure 10 shows the obtained results from the simulation.

As it can be seen from Figure 10, both set-based and proposed interval observer approaches are computing the same state estimation. There exists a small difference between the computed bounds. Looking at the simulation, the set-based interval observer approach is a bit more conservative than the proposed interval observer approach since the interval hull of state-bounding zonotope is used instead of the exact zonotope. 

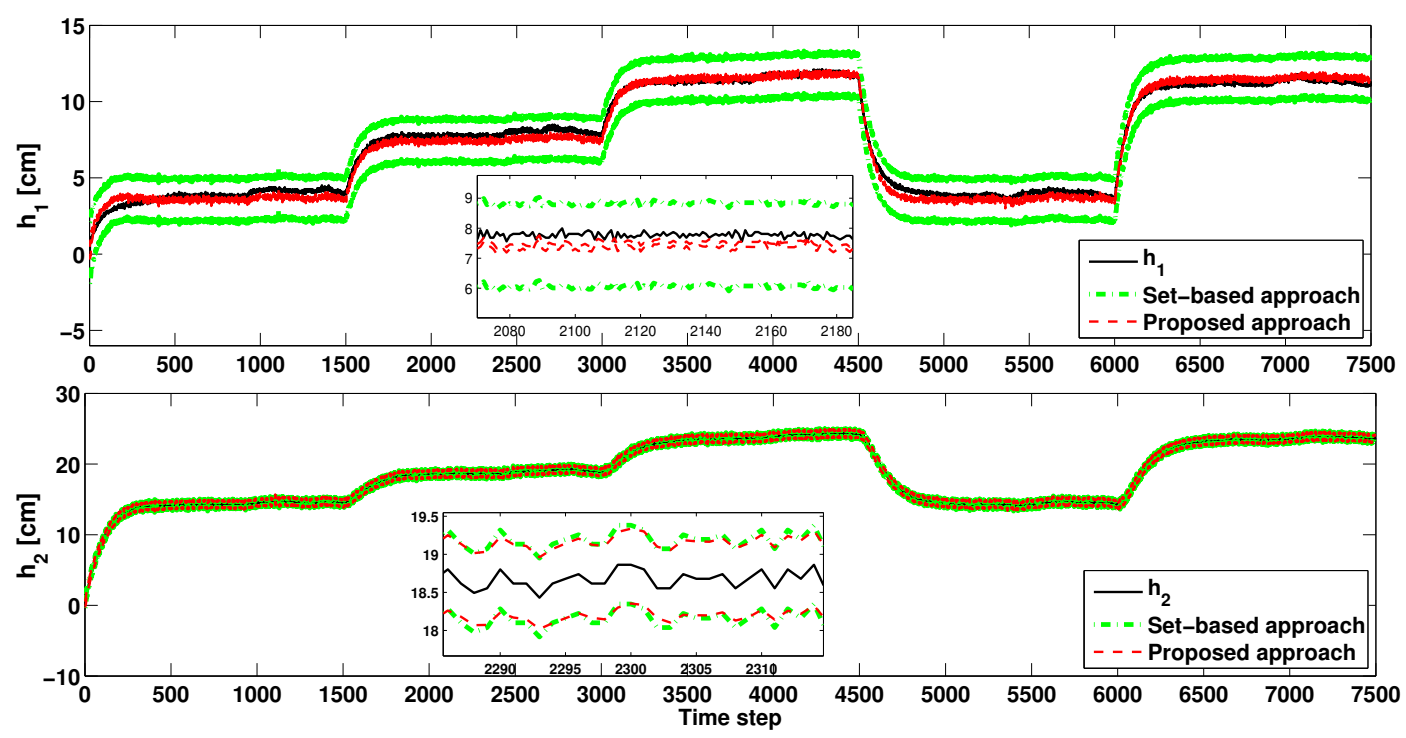

Figure 9. Set-based vs. proposed interval observer approaches considering monotonic system and nonmonotonic observer.

Moreover, similar to analysis of the example in Section 6.1, the new observer structure in Theorem 5.3 is also tested for the real case study. Figure 11 shows the obtained results from the simulation.

As it can be seen in Figure 11, set-based, proposed and the integrated interval observer approaches are compared for the case study. Since the considered case study is monotonic, i.e., all the elements of matrix $A$ are positive, the obtained results from the different observers are the same. It is worth mentioning that the observer gain in the set-based interval observer approach is computed by considering the satisfaction of the LMIs in Theorems 3.4 and 3.5 leading to $L=\left[\begin{array}{ll}0.0100 & 0.9903\end{array}\right]^{\top}$. Considering the proposed interval observer approach, the observer gain is designed using the LMIs in (54) and (55). In this case, $\bar{L}=\underline{L}=\left[\begin{array}{ll}0.001 & 0.9803\end{array}\right]^{\top}$. Furthermore, observer gain in the proposed integrated observer structure is calculated using Theorem 3.4 to satisfy the $H_{\infty}$ performance together with the LMI mentioned in (65) to satisfy the monotonicity property of the observer yielding to $L=\left[\begin{array}{ll}0.001 & 0.9803\end{array}\right]^{\top}$. The results obtained in Figure 11 show that the monotonicity and the convergence of the observers can be guaranteed for all different observers and they can compute almost the same results.

Therefore, both application examples illustrate that having a monotonic observer, almost the same results can be obtained by using the proposed LMIs to design the setbased and proposed interval observer approaches and it is well suited to also address the time-varying and time-invariant uncertainties. Furthermore, using the relationship between the set-based and proposed interval observer approaches, the new interval observer can be proposed based on the interval hull of the state-bounding zonotope where both problems of the set-based interval observer approach, i.e., preserving time dependency of the uncertain parameter and wrapping effect, are handled in non-monotonic systems. 


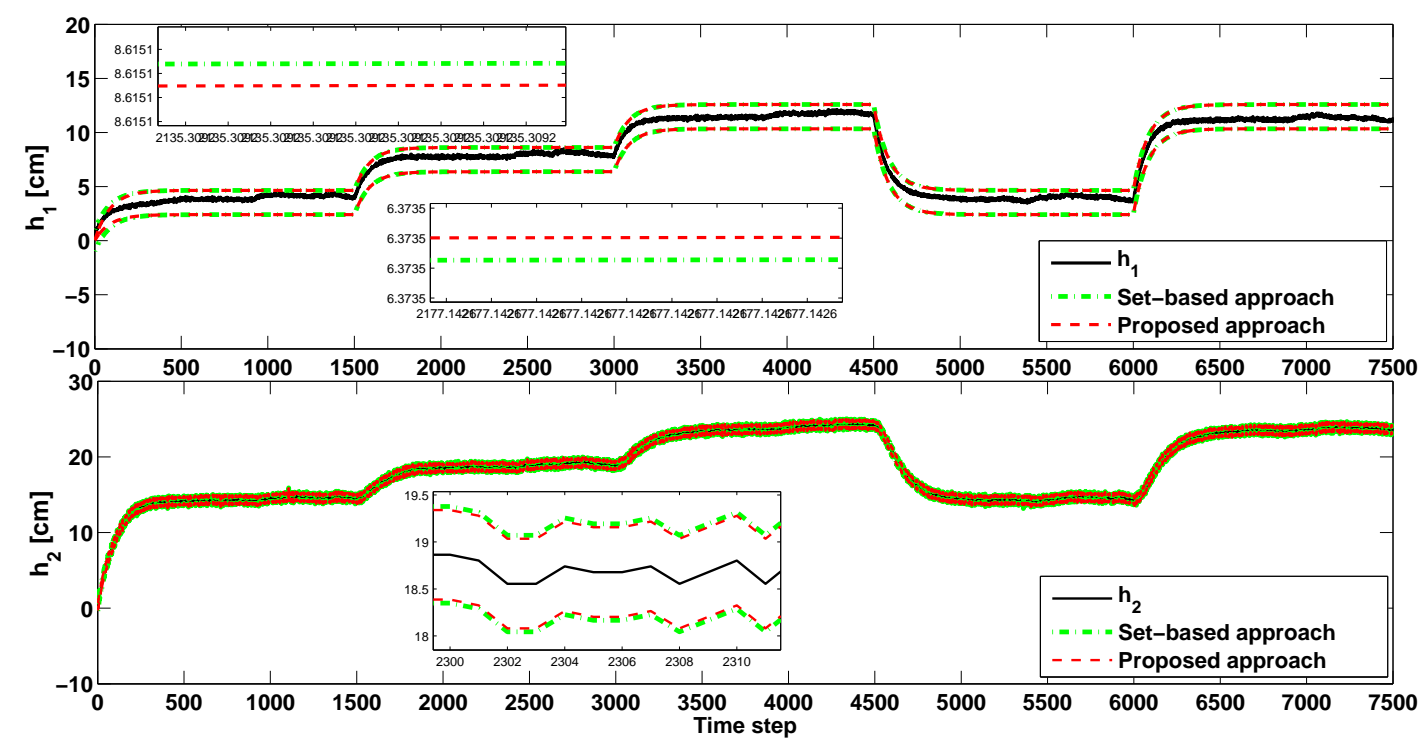

Figure 10. Set-based vs. proposed interval observer approaches considering monotonic system and monotonic observer.

\section{Conclusion}

This paper has proposed the design of an interval observer-based approach for discretetime linear systems with both time-invariant and time-varying uncertainties. First, the time-varying approach, called set-based interval observer approach, is introduced. In the set-based interval observer approach, $H_{\infty}$ performance and minimization of the size of the obtained state-bounding zonotope are considered to derive the LMI for computing the observer gain. Then, it is shown that in the set-based interval observer approach, the time dependency of the parameter uncertainties cannot be preserved. Furthermore, the wrapping effect problem appears when considering a non-monotonic system for the case of set-based interval observer approach. So far, in order to solve the issues of using the set-based interval observer approach, the time-invariant approach, called trajectory-based interval observer approach, is used to propose a new interval observer structure. The LMI technique is utilized in the proposed interval observer approach to guarantee the computation of the observer gain in order to satisfy both the $H_{\infty}$ performance and monotonicity property. As a novelty, based on the comparison of the mentioned interval observation approaches, it is shown that, using the interval hull of the state-bounding zonotope in set-based interval observer approach, both approaches can be connected when satisfying the monotonicity property. Furthermore, a method for designing the observer that can connect both type of interval observations is proposed. It is shown that the proposed approach, which is based on the set-based interval observer approach, has the same performance of the proposed interval observer approach to preserve the time dependency without having the problem of wrapping effect. The comparison of the set-based, proposed and the integrated interval observer approaches has been conducted on two different case studies, i.e., a numerical example and a real case study based on two-tank system. The obtained results from both case studies are well suited to address the results in the theoretical part of the paper. As a future research, the effectiveness of the proposed results will be investigated for enhancing the sensitivity to faults, rather than only the robust state estimation. 


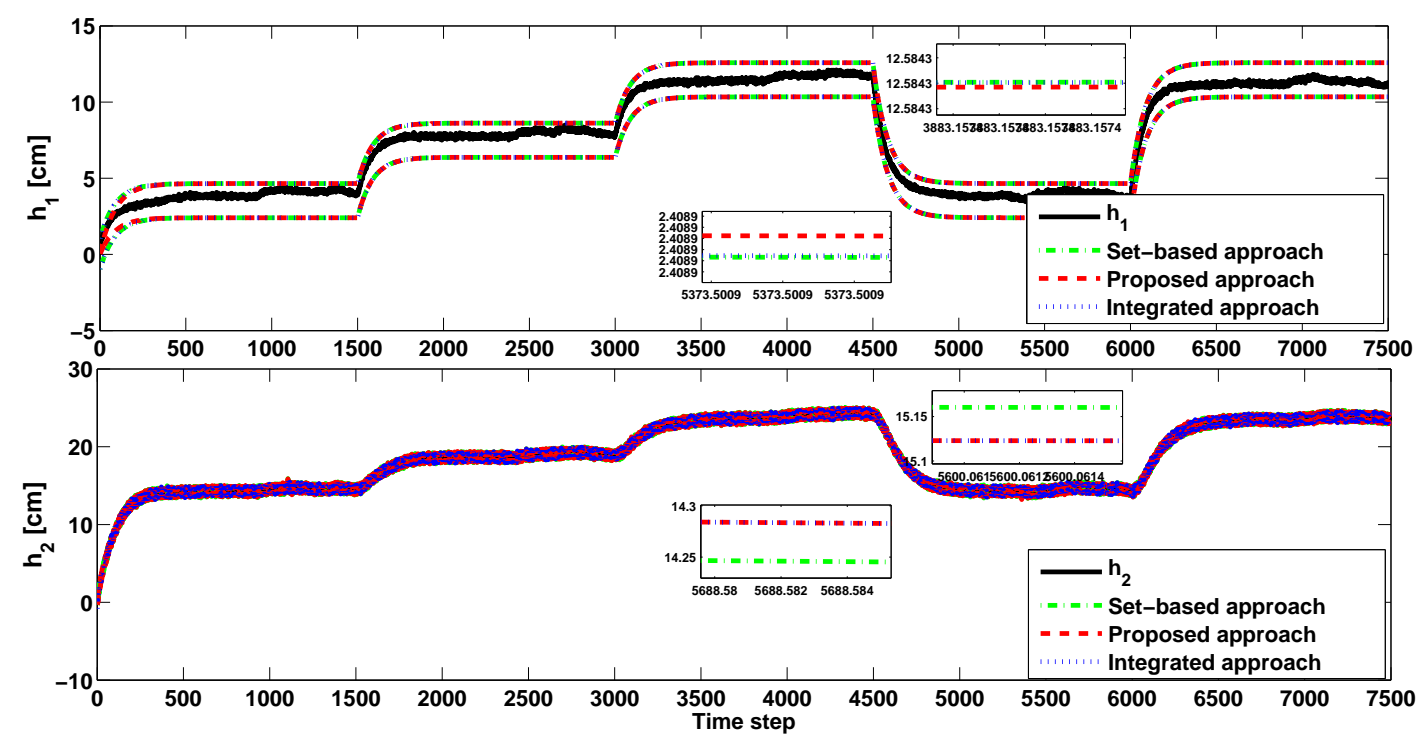

Figure 11. Set-based vs. proposed interval observer approaches considering monotonic system and designing the integrated monotonic observer.

\section{Appendix}

Definition 1. (Zonotope) An $m$-order zonotope $\mathcal{Z}=\left\langle c_{z}, R_{z}\right\rangle \in \mathbb{R}^{n}(m \geq n)$ is defined by a hypercube $\mathbf{B}^{m}=[-1,1]^{m}$ affine projection with the center $c_{z} \in \mathbb{R}^{n}$ and the shape matrix $R_{z} \in \mathbb{R}^{n \times m}$ as

$$
\mathcal{Z}=\left\langle c_{z}, R_{z}\right\rangle=\left\{c_{z}+R_{z} z, z \in \mathbf{B}^{m}\right\}
$$

Definition 2. (Minkowski sum) Considering two sets $\mathcal{A}$ and $\mathcal{B}$, their Minkowski sum is a set defined as $\mathcal{A} \oplus \mathcal{B}=\{a+b \mid a \in \mathcal{A}, b \in \mathcal{B}\}$. Furthermore, the Minkowski sum of the zonotopes $\mathcal{Z}_{1}=\left\langle c_{z_{1}}, R_{z_{1}}\right\rangle$ and $\mathcal{Z}_{2}=\left\langle c_{z_{2}}, R_{z_{2}}\right\rangle$ is $\mathcal{Z}_{1} \oplus \mathcal{Z}_{2}=\left\langle c_{z_{1}}+c_{z_{2}},\left[R_{z_{1}}, \quad R_{z_{2}}\right]\right\rangle$.

Definition 3. ( $F_{W}$-radius) Considering a weighting matrix $W \in \mathbb{R}^{n_{x} \times n_{x}}, W=W^{\top}>$ 0 , the weighted Frobenius radius of a given zonotope $Z=\langle c, R\rangle$ is defined using the weighted Frobenius norm of $R$, i.e., $\iota_{F, w}=\|\mathcal{Z}\|_{F, W}=\|R\|_{F, W}$.

Definition 4. ( $W$-radius) Considering a weighting matrix $W \in \mathbb{R}^{n_{x} \times n_{x}}, W=W^{\top}>$ 0 , the $W$-radius of a given zonotope $Z=\langle c, R\rangle$ with $R \in \mathbb{R}^{n_{x} \times r}$ is defined as $\iota_{w}=$ $\max _{z \in \mathcal{Z}}\|z-c\|_{2, W}^{2}=\max _{b \in \mathbf{B}^{r}}\|R b\|_{2, W}^{2}$ where $b$ is unitary box.

Definition 5. (Interval hull) (Le, Stoica, Alamo, Camacho, \& Dumur, 2013) The interval hull of a given zonotope $\mathcal{Z}=\left\langle c_{z}, R_{z}\right\rangle$ is the smallest interval box that contains $\mathcal{Z}$ and it is denoted by $\square \mathcal{Z}$ that can be computed by

$$
\square \mathcal{Z}=\left\{z:\left|z_{i}-c_{z_{i}}\right| \leq\left\|R_{z_{i}}\right\|_{1}\right\}
$$

where $R_{z_{i}}$ is the $i$-th row of $R_{z}$, and $c_{z_{i}}$ and $z_{i}$ denote the $i$-th components of $c_{z}$ and $z$, respectively. 
Property 1. (Linear image) The linear image of a zonotope $\mathcal{Z}=\langle c, R\rangle$ by a compatible matrix $L$ is $L \odot\langle c, R\rangle=\langle L c, L R\rangle$.

Property 2. (Reduction operator) A reduction operator denoted $\downarrow_{q}$ permits to reduce the number of generators of a zonotope $\langle c, R\rangle$ to a fixed number $q$ while preserving the inclusion property $\langle c, R\rangle \subset\left\langle c, \downarrow_{q}\{R\}\right\rangle$. A simple yet efficient solution to compute $\downarrow_{q}\{R\}$ is given in (Combastel, 2003). It consists in sorting the columns of $R$ on decreasing Euclidean norm and enclosing the influence of the smaller columns only into an easily computable interval hull, so that the resulting matrix $\downarrow_{q}\{R\}$ has no more than $q$ columns.

Property 3. (Zonotope inclusion) (Alamo et al., 2005) Given a zonotope $\mathcal{Z}=$ $\langle c, R\rangle \subset \mathbb{R}^{n}$, with a vector $c \in \mathbb{R}^{n}$ denoting the center and an interval matrix ${ }^{4}$ $R \in \mathbb{R}^{2(n \times m)}(n \leq m)$ denoting the shape of the zonotope, a zonotope inclusion indicated by $\diamond(\mathcal{Z})$ is defined as $\diamond(\mathcal{Z})=\langle c,[\operatorname{mid}(R) \quad S]\rangle$, where $S$ is a diagonal matrix that satisfies $S_{i i}=\sum_{j=1}^{m} \frac{\operatorname{diam}\left(R_{i j}\right)}{2}, i=1,2, \cdots, n$, with $\operatorname{mid}($.$) and \operatorname{diam}($.$) are the$ center and diameter of interval matrix, respectively.

Property 4. (State zonotope inclusion) (Guerra, Puig, \& Witczak, 2008) Given $\mathcal{X}_{k+1}=A \mathcal{X}_{k} \oplus B u_{k}$, where $A$ and $B$ are interval matrices and $u_{k}$ is the input at time instant $k$, considering $\mathcal{X}_{k}$ as a zonotope with the center $c_{x, k}$ and the shape ma$\operatorname{trix} R_{x, k}$ such $\mathcal{X}_{k}=\left\langle c_{x, k}, R_{x, k}\right\rangle$, the zonotopic state at the next time instant $k+1$ defined as $\mathcal{X}_{k+1}$ is bounded by a zonotope $\mathcal{X}_{k+1}^{e}=\left\langle c_{x, k+1}, R_{x, k+1}\right\rangle$, with

$$
\begin{aligned}
c_{x, k+1} & =\operatorname{mid}(A) c_{x, k}+\operatorname{mid}(B) u_{k}, \\
R_{x, k+1} & =\left[\begin{array}{lll}
\left(A R_{x, k}\right) & \frac{\operatorname{diam}(A)}{2} c_{x, k} & \frac{\operatorname{diam}(B)}{2} u_{k}
\end{array}\right],
\end{aligned}
$$

where $\diamond\left(A R_{x, k}\right)$ shows the shape matrix of the state-bounding zonotope.

Property 5. (Monotonicity property) If the variation of the state function regarding to all the states and parameters is positive, the discrete time system will satisfy the monotonicity property. Moreover, those systems that are satisfied this property are the monotonic systems.

Lemma 1. Based on Efimov, Raïssi, Perruquetti, E Zolghadri (2013), if $\underline{A} \leq A \leq \bar{A}$ for $\underline{A}, A, \bar{A} \in \mathbb{R}^{n_{x} \times n_{x}}$ and $\underline{x} \leq x \leq \bar{x}$ for $\underline{x}, x, \bar{x} \in \mathbb{R}^{n_{x}}$, then

$$
\underline{A^{+}} \underline{x^{+}}-\overline{A^{+}} \underline{x^{-}}-\underline{A^{-} \overline{x^{+}}}+\overline{A^{-}} \overline{x^{-}} \leq A x \leq \overline{A^{+}} \overline{x^{+}}-\underline{A^{+}} \overline{x^{-}}-\overline{A^{-}} \underline{x^{+}}+\underline{A^{-}} \underline{x^{-}},
$$

where $\overline{A^{+}}=\max \{0, \bar{A}\}, \overline{A^{-}}=\overline{A^{+}}-\bar{A}, \underline{A^{+}}=\max \{0, \underline{A}\}, \underline{A^{-}}=\underline{A^{+}}-\underline{A}, \overline{x^{+}}=$ $\max \{0, \bar{x}\}, \overline{x^{-}}=\overline{x^{+}}-\bar{x}, \underline{x^{+}}=\max \{0, \underline{x}\}$ and $\underline{x^{-}}=\underline{x^{+}}-\underline{x}$.

Lemma 2. Given a system dynamic that is considered to be function of the parameter vectors as

$$
x_{k+1}=A(\theta) x_{k}+B(\theta) u_{k},
$$

\footnotetext{
${ }^{4} \mathrm{An}$ interval matrix is a matrix whose elements are interval numbers (Ganesan, 2007).
} 
where $A$ and $B$ are known, $\Delta A(\theta)$ and $\Delta B(\theta)$ are the uncertain terms with considering the Assumption 2.1, i.e., $\underline{\Delta A}<\Delta A(\theta)<\overline{\Delta A}$ and $\underline{\Delta B}<\Delta B(\theta)<\overline{\Delta B}$. Then it can be written that

$$
\begin{aligned}
& \overline{A(\theta)}=A+\overline{\Delta A(\theta)}, \\
& \underline{\overline{A(\theta)}}=A+\underline{\overline{B(\theta)}}=B+\overline{\overline{\Delta B(\theta)}}, \\
& \underline{B(\theta)}=B+\underline{\Delta B(\theta) .}
\end{aligned}
$$

Moreover, by assuming that $\Delta A(\theta)$ and $\Delta B(\theta)$ satisfy the following match perturbation condition:

$$
\begin{aligned}
& {\left[\begin{array}{ll}
\overline{\Delta A(\theta)} & \overline{\Delta B(\theta)}
\end{array}=E_{\theta} \bar{\Xi}\left[\begin{array}{ll}
G_{a} & G_{b}
\end{array}\right],\right.} \\
& {\left[\begin{array}{ll}
\Delta A(\theta) & \underline{\Delta B(\theta)}
\end{array}\right]=E_{\theta} \Xi\left[\begin{array}{ll}
G_{a} & G_{b}
\end{array}\right],}
\end{aligned}
$$

where $\bar{\Xi}$ and $\Xi$ are the block diagonal matrices which represents the parameter uncertainties for the upper and lower bounds, and, $E_{\theta}$ is the associated distribution matrix with appropriate dimensions. Furthermore, $G_{a}$ and $G_{b}$ are known matrices. In this case, if the parameter vector is perturbed around the nominal value $\theta=\theta_{0}$, the system can be rewritten as

$$
\begin{aligned}
& \bar{x}_{k+1}=A\left(\theta_{0}\right) \bar{x}_{k}+B\left(\theta_{0}\right) u_{k}+E_{\theta} \bar{\theta}_{k}, \\
& \underline{x}_{k+1}=A\left(\theta_{0}\right) \underline{x}_{k}+B\left(\theta_{0}\right) u_{k}+E_{\theta} \underline{\theta}_{k},
\end{aligned}
$$

where

$$
\begin{aligned}
E_{\theta} & =\left[\begin{array}{lll}
\frac{\partial A}{\partial \theta_{0}} & \mid & \frac{\partial B}{\partial \theta_{0}}
\end{array}\right], \\
\bar{\theta}_{k} & =\left[\begin{array}{l|lll|l|l}
\delta \bar{\theta}_{1} x_{k}^{\top} & \delta \bar{\theta}_{1} u_{k}^{\top} & \ldots & \delta \bar{\theta}_{N} x_{k}^{\top} & \delta \bar{\theta}_{N} u_{k}^{\top}
\end{array}\right], \\
\underline{\theta}_{k} & =\left[\begin{array}{lllllll}
\delta \underline{\theta}_{1} x_{k}^{\top} & \delta \underline{\theta}_{1} u_{k}^{\top} & \ldots & \delta \underline{\theta}_{N} x_{k}^{\top} & \delta \underline{\theta}_{N} u_{k}^{\top}
\end{array}\right] .
\end{aligned}
$$

Proof. According to Chen \& Patton (2012), the parameter perturbation can be approximated as

$$
\begin{aligned}
& \overline{\Delta A(\theta)} \approx \sum_{i=1}^{N} \overline{a_{i}(\theta)} A_{i}, \\
& \underline{\Delta A(\theta)} \approx \sum_{i=1}^{N} \underline{a_{i}(\theta)} A_{i}, \\
& \overline{\Delta B(\theta)} \approx \sum_{i=1}^{N} \overline{b_{i}(\theta)} B_{i}, \\
& \underline{\Delta B(\theta)} \approx \sum_{i=1}^{N} \underline{b_{i}(\theta)} B_{i},
\end{aligned}
$$

where $A_{i}$ and $B_{i}$ are the constant matrices and $a_{i}$ and $b_{i}$ are unknown scalar vectors. 
Then, considering (76) and (80), the uncertain parameter can be approximated only based on the disturbance term as

$$
\begin{gathered}
E_{\theta} \bar{\theta}_{k}=\overline{\Delta A(\theta)} \bar{x}_{k}+\overline{\Delta B(\theta)} u_{k}=\left[\begin{array}{llllll}
A_{1} & \ldots & A_{N} & B_{1} & \ldots & B_{N}
\end{array}\right]\left[\begin{array}{c}
\overline{a_{1}(\theta)} \bar{x}_{k} \\
\frac{\ldots}{a_{N}(\theta)} \bar{x}_{k} \\
\overline{b_{1}(\theta)} u_{k} \\
\ldots \\
\overline{b_{N}(\theta)} u_{k}
\end{array}\right], \\
E_{\theta} \underline{\theta}_{k}=\underline{\Delta A(\theta)} \underline{x}_{k}+\underline{\Delta B(\theta)} u_{k}=\left[\begin{array}{llllll}
A_{1} & \ldots & A_{N} & B_{1} & \ldots & B_{N}
\end{array}\right]\left[\begin{array}{c}
\frac{a_{1}(\theta)}{\ldots} \\
\frac{a_{N}(\theta)}{x_{k}} \\
\frac{b_{1}(\theta)}{\ldots} u_{k} \\
\underline{b_{N}(\theta)} u_{k}
\end{array}\right],
\end{gathered}
$$

where $\theta$ shows the disturbance term. Moreover, by assuming (77), $\phi_{k}$ can be considered as

$$
\begin{aligned}
\bar{\theta}_{k} & =\bar{\Xi}_{k}\left[\begin{array}{ll}
G_{a} \bar{x}_{k} & G_{b} u_{k}
\end{array}\right], \\
\underline{\theta}_{k} & =\Xi_{k}\left[\begin{array}{ll}
G_{a} \underline{x}_{k} & G_{b} u_{k}
\end{array}\right] .
\end{aligned}
$$

Thus, if the system is considered to be function of the parameter vectors where the parameter vector is perturbed around the nominal value $\theta=\theta_{0}$ as the dynamical model in (75), it can be written that

$$
\begin{aligned}
& \bar{x}_{k+1}=A\left(\theta_{0}\right) \bar{x}_{k}+B\left(\theta_{0}\right) u_{k}+\sum_{i=1}^{N}\left\{\frac{\partial A}{\partial \theta_{0}} \delta \bar{\theta}_{i} \bar{x}_{k}+\frac{\partial B}{\partial \theta_{0}} \delta \bar{\theta}_{i} u_{k}\right\}, \\
& \underline{x}_{k+1}=A\left(\theta_{0}\right) \underline{x}_{k}+B\left(\theta_{0}\right) u_{k}+\sum_{i=1}^{N}\left\{\frac{\partial A}{\partial \theta_{0}} \delta \underline{\theta}_{i} \underline{x}_{k}+\frac{\partial B}{\partial \theta_{0}} \delta \underline{\theta}_{i} u_{k}\right\} .
\end{aligned}
$$

Therefore, considering (83), the dynamical model in (75) can be rewritten as it is characterized in (78).

\section{Acknowledgement(s)}

This work has been partially funded by the Spanish Government (MINECO) through the project DEOCS (ref. DPI2016-76493-C3-3-R), by MINECO and FEDER through the project HARCRICS (ref. DPI2014-58104-R) and by Agència de Gestió d'Ajuts Universitaris i de Recerca. Furthermore, the authors thank Brais Gonzàlez García for his contribution in designing the real experiments.

\section{References}

Alamo, T., Bravo, J. M., \& Camacho, E. F. (2005). Guaranteed state estimation by zonotopes. Automatica, 41(6), 1035-1043. 
Blanke, M., Kinnaert, M., Lunze, J., Staroswiecki, M., \& Schröder, J. (2006). Diagnosis and fault-tolerant control (Vol. 691). Springer.

Chen, J., \& Patton, R. J. (2012). Robust model-based fault diagnosis for dynamic systems (Vol. 3). Springer Science \& Business Media.

Combastel, C. (2003). A state bounding observer based on zonotopes. In European Control Conference (ECC), UK (pp. 2589-2594).

Combastel, C. (2015). Zonotopes and kalman observers: Gain optimality under distinct uncertainty paradigms and robust convergence. Automatica, 55, 265-273.

Cugueró, P., Puig, V., Saludes, J., \& Escobet, T. (2002). A class of uncertain linear interval models for which a set based robust simulation can be reduced to few pointwise simulations. In Proceedings of the 41st ieee conference on decision and control, 2002. (Vol. 2, pp. 1862 1863).

Ding, S. X. (2008). Model-based fault diagnosis techniques: design schemes, algorithms, and tools. Springer Science \& Business Media.

Efimov, D., Raïssi, T., Perruquetti, W., \& Zolghadri, A. (2013). Estimation and control of discrete-time LPV systems using interval observers. In IEEE 52nd Annual Conference on Decision and Control (CDC), Italy (pp. 5036-5041).

Efimov, D., Raïssi, T., \& Zolghadri, A. (2013). Control of nonlinear and LPV systems: interval observer-based framework. IEEE Transactions on Automatic Control, 58(3), 773-778.

El Ghaoui, L., \& Calafiore, G. (2000). Identification of arx models with time-varying bounded parameters: A semidefinite programming approach. IFAC Proceedings Volumes, 33(15), 283-288.

Ganesan, K. (2007). On some properties of interval matrices. International Journal of Mathematics Sciences, $1,2$.

Gertler, J. (1997). Fault detection and isolation using parity relations. Control Engineering Practice, 5(5), 653-661.

Guerra, P., Puig, V., \& Witczak, M. (2008). Robust fault detection with unknown-input interval observers using zonotopes. IFAC Proceedings Volumes, 41(2), 5557-5562.

Horak, D. T. (1988). Failure detection in dynamic systems with modeling errors. Journal of Guidance, Control, and Dynamics, 11(6), 508-516.

Kalman, R. E. (1960). A new approach to linear filtering and prediction problems. Journal of Basic Engineering, 82(1), 35-45.

Karimi Pour, F., Puig, V., \& Ocampo-Martinez, C. (2017). Comparative assessment of LPVbased predictive control strategies for a pasteurization plant. In 4th International Conference on Control, Decision and Information Technologies, Spain (pp. 1-6).

Kolev, L., \& Petrakieva, S. (2005). Assessing the stability of linear time-invariant continuous interval dynamic systems. IEEE Transactions on Automatic Control, 50(3), 393-397.

Le, V. T. H., Alamo, T., Camacho, E. F., Stoica, C., \& Dumur, D. (2012). Zonotopic setmembership estimation for interval dynamic systems. In American Control Conference (ACC), Canada (pp. 6787-6792).

Le, V. T. H., Stoica, C., Alamo, T., Camacho, E. F., \& Dumur, D. (2013). Zonotopes: From guaranteed state-estimation to control. John Wiley \& Sons.

Maybeck, P. S. (1982). Stochastic models, estimation, and control (Vol. 3). Academic Press, USA.

Montes de Oca, S., Puig, V., \& Blesa, J. (2012). Robust fault detection based on adaptive threshold generation using interval LPV observers. International Journal of Adaptive Control and Signal Processing, 26(3), 258-283.

Nam, P. T., Pathirana, P. N., \& Trinh, H. (2014). $\varepsilon$-bounded state estimation for time-delay systems with bounded disturbances. International Journal of Control, 87(9), 1747-1756.

Nam, P. T., Trinh, H. M., \& Pathirana, P. N. (2016). Componentwise ultimate bounds for positive discrete time-delay systems perturbed by interval disturbances. Automatica, 72, 153-157.

Pourasghar, M., Puig, V., Ocampo-Martinez, C., \& Zhang, Q. (2017). Reduced-order intervalobserver design for dynamic systems with time-invariant uncertainty. IFAC-PapersOnLinen, 
France, 50(1), 6271-6276.

Puig, V., Cugueró, P., \& Quevedo, J. (2001). Worst-case state estimation and simulation of uncertain discrete-time systems using zonotopes. In 2001 european control conference (ecc) (pp. 1691-1697).

Puig, V., Montes de Oca, S., \& Blesa, J. (2013). Adaptive threshold generation in robust fault detection using interval models: time-domain and frequency-domain approaches. International Journal of Adaptive Control and Signal Processing, 27(10), 873-901.

Puig, V., Saludes, J., \& Quevedo, J. (1999). A new algorithm for adaptive threshold generation in robust fault detection based on a sliding window and global optimization. In European Control Conference (ECC), germany (pp. 1546-1551).

Puig, V., Saludes, J., \& Quevedo, J. (2003). Worst-case simulation of discrete linear timeinvariant interval dynamic systems. Reliable Computing, 9(4), 251-290.

Puig, V., Stancu, A., \& Quevedo, J. (2005a). Observers for interval systems using set and trajectory-based approaches. In 44th IEEE Conference on Decision and Control and European Control Conference, Spain (pp. 6567-6572).

Puig, V., Stancu, A., \& Quevedo, J. (2005b). Simulation of uncertain dynamic systems described by interval models: A survey. IFAC Proceedings Volumes, Czech Republic, 38(1), 1239-1250.

Raïssi, T., Efimov, D., \& Zolghadri, A. (2012). Interval state estimation for a class of nonlinear systems. IEEE Transactions on Automatic Control, 57(1), 260-265.

Sadrnia, M., Chen, J., \& Patton, R. (1996). Robust fault diagnosis observer design using $H_{\infty}$ optimisation and $\mu$ synthesis. IEEE Colloquium on Modelling and Signal Processing for Fault Diagnosis, 1-9.

Schweppe, F. (1968). Recursive state estimation: Unknown but bounded errors and system inputs. IEEE Transactions on Automatic Control, 13(1), 22-28.

Tibken, B. (1993). A new simulation tool for uncertain discrete time systems. In European Control Conference (ECC), netherlands.

Wang, Y., Wang, Z., Puig, V., \& Cembrano, G. (2018). Zonotopic set-membership state estimation for discrete-time descriptor LPV systems. IEEE Transactions on Automatic Control.

Wang, Y., Zhou, M., Puig, V., Cembrano, G., \& Wang, Z. (2017). Zonotopic fault detection observer with h- performance. In 36th chinese control conference (ccc), China (pp. 7230 $7235)$.

Zhang, Y., \& Jiang, J. (2008). Bibliographical review on reconfigurable fault-tolerant control systems. Annual Reviews in Control, 32(2), 229-252.

Zhong, M., Ding, S. X., Lam, J., \& Wang, H. (2003). An LMI approach to design robust fault detection filter for uncertain LTI systems. Automatica, 39(3), 543-550. 